\title{
World Congress on Osteoporosis, Osteoarthritis and Musculoskeletal Diseases (WCO-IOF-ESCEO 2020): Non-Sponsored Symposia Abstracts
}

(C) International Osteoporosis Foundation and National Osteoporosis Foundation 2020

\section{NSS1 \\ GUIDELINES FOR HORMONAL APPROACH ON TRANSGENDER PATIENTS \\ A. Sierra Osorio $^{1}$ \\ ${ }^{1}$ Internist and endocrinologist, Coordinator of gender disphoria program, Hospital San Jose, Bogotá, Colombia}

Gender dysphoria refers to the condition in which a person experiences incongruence between their gender identity and the biological sex designated at birth, leading them to persistent discomfort with the expected social role and functional deterioration in different areas of daily life (1). In adults, it is defined by the criteria included in the DSM-V manual (2) (3). They are categorized as transgender women or trans / female women (change from man to woman: $\mathrm{MtF} /$ man to woman) and transgender men or trans man / man (change from woman to man: FtM / woman to man) (1) (4). With respect to the prevalence of gender dysphoria, the data varies in the publications over time (changes in the definition and criteria of diagnosis varies over time), however, a systematic review and meta-analysis of observational studies conducted between 1945 and 2014, of which 85.7\% were conducted in Europe, found a global prevalence of 4.6 in 100,000 individuals, 6.8 and 2.6 for transgender women and men, respectively (5). Once the diagnosis is confirmed, the process of gender affirming therapies can be initiated ideally in a center that has multidisciplinary care (Endocrinology, Psychiatry, Urology, Gynecology, Plastic Surgery) and management experience $(2,4)$. The gender affirmation process is achieved through several steps that include: continuous support therapy by Psychiatry, use of hormonal therapy and, in some cases, gender affirmation surgery (2). The goal of gender affirmation hormone therapy is to reduce endogenous sex hormone levels and secondary sexual characteristics of biological sex and replace hormone levels consistent with gender identity, mitigating discomfort related to incongruity. (6). For hormonal therapy, different schemes are used (dose, administration and periodicity) that are chosen according to the individual characteristics and preferences of the patients, testosterone (FtM) and estrogens (in association or not with antidrogenic agents) (MtF) being the most used medications. Periodic follow-up sessions include anamnesis, exploration of physical signs of virilization/feminization, serum hormone levels and surveillance of complications associated with hormone administration (4) (6) (7) (8). Through the measurement of testosterone and estrogen levels, the biochemical response to hormonal treatment is evaluated. In transgender women, the biochemical goal is obtained with levels of total testosterone $<50 \mathrm{ng} / \mathrm{dl}$ and serum estradiol in the normal physiological range of cisgender women (between 20-200 pg / $\mathrm{ml}$ ) and for the group of transgender men with a level of estradiol $<50 \mathrm{pg} / \mathrm{ml}$ and total testosterone levels within the physiological range corresponding to cis men (350$700 \mathrm{ng} / \mathrm{dl}$ ) (6). The intentional use of supraphysiological doses or the use of inadequate doses to maintain the serum concentration of crossed sex hormones within the physiological range of the gender affirmed, may constitute a risk factor for the development of some complications, such as osteoporosis (especially in cases of patients with genital affirmation surgery / gonadectomy and who have suspended the affirmative hormonal substitution), increased cardiovascular risk, thromboembolic disease, increased risk of cancer, among others. (2) (6) (9)

\section{References}

1. Ahmad S, Barrett J, Beaini AY, Bouman WP, Davies A, Greener HM, et al. Gender dysphoria services: a guide for general practitioners and other healthcare staff. Sexual and Relationship Therapy. 2013;28(3):172-85.

2. Hembree WC, Cohen-Kettenis P, Delemarre-van de Waal HA, Gooren LJ, Meyer WJ, Spack NP, et al. Endocrine treatment of transsexual persons: an Endocrine Society clinical practice guideline. J Clin Endocrinol Metab. 2009;94(9):3132-54.

3. American Psychiatric A. Diagnostic and statistical manual of mental disorders (DSM-5®): American Psychiatric Pub; 2013 
4. Wylie K, Barrett J, Besser M, Bouman WP, Bridgman M, Clayton A, et al. Good practice guidelines for the assessment and treatment of adults with gender dysphoria. Sexual and Relationship Therapy. 2014;29(2):154-214

5. Arcelus J, Bouman WP, Van Den Noortgate W, Claes L, Witcomb G, Fernandez-Aranda F. Systematic review and meta-analysis of prevalence studies in transsexualism. Eur Psychiatry. 2015;30(6):807-15

6. Hembree WC, Cohen-Kettenis PT. Endocrine Treatment of Gender-Dysphoric/ Gender-Incongruent Persons: An Endocrine Society. Clinical Practice GuidelineJ Clin Endocrinol Metab, November 2017, 102(11):3869-3903

7. Irwig MS. Testosterone therapy for transgender men. Lancet Diabetes Endocrinol. 2017;5(4):301-11.

8. Tangpricha V, den Heijer M. Oestrogen and antiandrogen therapy for transgender women. Lancet Diabetes Endocrinol. 2017;5(4):291-300.

9. Asscheman H, Giltay EJ, Megens JA, de Ronde WP, van Trotsenburg MA, Gooren LJ. A long-term follow-up study of mortality in transsexuals receiving treatment with crosssex hormones. Eur J Endocrinol. 2011;164(4):635-42.

\section{NSS2 \\ FROM MAN TO WOMAN AND FROM WOMAN TO MAN: DOES IT MATTER? \\ ${\text { A. } \text { Coy }^{1}}^{1}$ \\ ${ }^{1}$ Internist, Fellow in endocrinology, Fundacion Universitaria Ciencias de la Salud, Hospital San Jose, Bogotá, Colombia}

It has been described that as long as a transgender individual is on standard gender affirming hormone treatment bone mass density (BMD) should not decrease, however the opposite is true in case of periods of hypogonadism or bad compliance to hormone therapy (HT). The Z score of transgender individuals should be calculated using the gender conforming the individuals identity. (1). Androgens have a special role on trabecular bone health and both testosterone and estradiol are important for cortical bone health (3). Reports have discovered that transgender women have lower bone density compared to cisgender men before HT which has been hypothesized to be due to low physical activity, Vitamin D deficit and cigarette smoking $(1,2,3)$. In transgender women an statistical significant increase has been described on lumbar spine bone mass density (BMD), to opposite is true for hip BMD (2). In transgender men similar bone composition at baseline has been found compared to cisgender women. Hormone therapy has shown to preserve or increase bone density which hypothesized to be due to the aromatization of testosterone to estradiol, which has been reported specially in transmen of postmenopausal age. In addition, there is an increase in both muscle mass and strength $(2,3,5)$. It is important to conclude that HT has not been associated with a negative effect on BMD, indicating bone safety in transgender individuals (4). The story is different in cohorts after gonadectomy where a decreased has been observed, which could be due to periods of hypogonadism (6).

\section{References}

1. Rosen, HN; Hamnvik, O-P R; Jaisamrarn U; Malabanan IO, Safer JD, Tangpricha V, Wattanachanya L, Swan S. Yeap. Bone densitometry in transgender and gender non conforming (TGNC) individuals: The 2019 ISCD official positions. Journal of Clinical Densitometry 2019; 22 (4): 544-53.

2. T'Sjoen, G; Arcelus, J; Gooren, L; et all. Endocrinology of transgender medicine. Endocrine Reviews 40:97-117,2019.

3. Davidge-Pitts, C; Clarke, B. Transgender bone health. Maturitas 127 (2019), 35-42.

4. Wiepjes, c; Jongh, R; de Blok, C; et all. Bone safety during the first ten years of gender affirming hormonal treatment in transwomen and transmen. Journal of Bone and Mineral Research, 2019, 447-454.

5. Wiepjes, C; Vlot, M; Klaver, M; et all. Bone mineral density increases in trans persons after 1 year of hormonal treatment: a multicenter prospective observational study. Journal of Bone and Mineral Research, June 2017, 1252-1260.

6. Dobrolinska, M; van der Tuuk, K; Vink, P; et all. Bone mineral density in transgender individuals after gonadectomy and long term gender affirming hormonal treatment. J Sex Med, 2019.

\section{NSS3}

\section{DENSITOMETRIC ACQUISITION, ANALYSIS AND} REPORTING

B. Muzzi Camargos ${ }^{1}$

${ }^{1}$ Clinical densitometryst, Bone densitometry and metabolism unit coordinator at Densimater, Rede Mater Dei, Belo Horizonte, Brazil

The International Society for Clinical Densitometry (ISCD) issued a Position Statement paper addressing steps to be followed once performing densitometric procedures that should be followed for the transgender patints. One of the recommedations is that the $\mathrm{T}$-scores should be used to diagnose osteoporosis, according to age. Routine baseline BMD testing is not indicated in transgender women, who have lower BMD than cisgender men in 3 months before receive gender affirming hormone treatment - GAHT - (1). Baseline BMD testing is indicated for TG women and men individuals if they have any of the following conditions: history of gonadectomy or therapy that lowers endogenous gonadal steroid levels prior to initiation of HT, hypogonadism with no plan to take GAHT, existing ISCD indications for BMD testing, such as glucocorticoid use and hyperparathyroidism (1). About T- and Z-Score calculation in TG individuals, T-scores should be calculated using a uniform Caucasian (nonrace adjusted) female normative database for all TG individuals of all ethnic groups; ISCD (2019) recommends using a T-score of $<2.5$ for diagnosis of 
osteoporosis in all TG individuals $>50$ years old, regardless of hormonal status. Z-scores should be calculated using the normative database that matches the gender identity of the individual according to the ISCD 2013 guidelines (2). Transgender men have been reported to have favorable BMD measurements compared to cisgender women (3). Other ISCD recommendations are to evaluate both transgenders - male and female - according to the Z-score derived from the cisgender when they have received at least 3 months of hormone therapy. Another very important point is that BMD testing should be done as closest as possible to gonadectomy (if present), and preferably prior to the initiation of hormone therapy (1). The male database is better for calculation of the $\mathrm{Z}$ score in transgender men because the BMD in transgender men is higher than in cisgender women; on the other hand, the review of the literature suggests that using the female database to calculate $\mathrm{Z}$ scores in transgender women is more valid than using the male database. The ideal situation would be to have a database of transgender women and men (2). Transgender male have a larger bone size and studies report a maintained BMD $(4,5)$.

\section{References}

1. Rosen, HN; Hamnvik, O-P R; Jaisamrarn U; Malabanan IO, Safer JD, Tangpricha V, Wattanachanya L, Swan S. Yeap. Bone densitometry in transgender and gender non conforming (TGNC) individuals: The 2019 ISCD official positions. Journal of Clinical Densitometry 2019; 22 (4): 544-53.

2. Watts NB, Leslie WD, Foldes AJ, Miller PD. 2013 International Society for Clinical Densitometry position development conference: task force on normative databases. J Clin Densitom 16(4):472-481.

3. Van Caenegem E, Wierckx K, Taes Y, et al. 2015 Body composition, bone turnover, and bone mass in trans men during testosterone treatment: 1-year follow-up data from a prospective case-controlled study (ENIGI). Eur J Endocrinol 172(2):163-171

4. T'sjoen, G; Arcelus, J; Gooren, I; et all. Endocrinology of transgender medicine. Endocrine reviews, februrary 2019, 40(1):97-117.

5. T'sjoen, G; Arcelus, J; Gooren, 1; et all. Endocrinology of transgender medicine. Endocrine reviews, endocrine society, 2018.

\section{NSS4}

\section{RECOMENDATIONS TO MAINTAIN BONE HEALTH ON TRANSGENDER PATIENTS}

\section{A. Medina Orjuella}

${ }^{1}$ Internist and endocrinologist coordinator of Osteoporosis and Fragility Fracture Program, Hospital San Jose, Associated Professor at Fundacion Universitaria Ciencia de la Salud, Bogotá, Colombia
The International Society of Clinical Densitometry (ISCD) published in 2109 , the recommendations in transgender (TG) patients based on indications for baseline Bone Mineral Density (BMD) testing, follow-up BMD testing and T- \& Z-Score calculation, FRAX and DXA reports parameters (1). The Follow-up BMD testing is not indicated in TG individuals on Hormone Therapy (HT) because gender affirming hormone treatment (GAHT) increases or maintains BMD unless there is low bone density as defined by current ISCD guidelines or another reason to suspect bone loss (treatment to suppress puberty, such as GnRH analogs, no therapeutic adherence or inadequate doses of GAHT, plan to discontinue GAHT, and presence of other risks for bone loss or fragility fracture). BMD testing intervals should be individualized (every 1- 2 yr until BMD is stable (1). Routine baseline BMD testing is not indicated in transgender women, who have lower BMD than cisgender men in 3 months before receive gender affirming hormone treatment (GAHT), maybe due to lower mean duration of sports activity than cisgender male controls, leading to lower muscle strength resulting in the lower BMD. Over the long term GAHT, the BMD is stable and there is no evidence of a higher imminent fracture risk $(2,3,4,5)$. On the other hand, transgender men have been reported to have favorable BMD measurements compared to cisgender women (6), and testosterone therapy increases or maintains BMD in transgender men, on a long time (4). For Fracture Risk Assessment Tool (FRAX) there is no scientific basis to recommend which gender to use in calculating fracture risk in TG individuals (7). The parameters to be included in the DXA report for transgender individuals are the same as are included in reports for the general population, but, the report should include both male and female Z-scores databases.(7)

\section{References}

- Rosen, HN; Hamnvik, O-P R; Jaisamrarn U; Malabanan IO, Safer JD, Tangpricha V, Wattanachanya L, Swan S. Yeap.. Bone densitometry in transgender and gender non conforming (TGNC) individuals: The 2019 ISCD official positions. Journal of Clinical Densitometry 2019; 22 (4): $544-53$.

- Fighera TM, da Silva E, Lindenau JD-R, Spritzer PM. 2018 Impact of cross-sex hormone therapy on bone mineral density and body composition in transwomen. Clin Endocrinol (Oxf) 88(6):856-862.

- Wierckx K, Van Caenegem E, Schreiner T, et al. 2014 Cross sex hormone therapy in trans persons is safe an effective at short time follow up: results from the European Network for the investigation of gender incongruence. J Sex Med 11(8):1999-2011

- Wiepjes CM, Vlot MC, Klaver M, et al. 2017 Bone mineral density increases in trans persons after 1 year of hormonal treatment: a multicenter prospective observational study. J Bone Miner Res 32(6):1252-1260 
- Wiepjes CM, de Jongh RT, de Blok CJ, et al. 2019 Bone safety during the first ten years of gender_affirming hormonal treatment in transwomen and transmen. J Bone Miner Res 34(3):447-454.

- Van Caenegem E, Wierckx K, Taes Y, et al. 2015 Body composition, bone turnover, and bone mass in trans men during testosterone treatment: 1-year follow-up data from a prospective case-controlled study (ENIGI). Eur J Endocrinol 172(2):163-171

- Watts NB, Leslie WD, Foldes AJ, Miller PD. 2013 International Society for Clinical Densitometry position development conference: task force on normative databases. J Clin Densitom 16(4):472-481.

\section{NSS5}

GLOBAL IMPACT OF BONE HEALTH TELEECHO: THE PROTOTYPE FOR TECHNOLOGY ENABLED COLLABORATIVE LEARNING FOR SKELETAL HEALTHCARE

E. M. Lewiecki $^{1}$

${ }^{1}$ University of New Mexico Health Sciences Center, Albuquerque, United States

Bone Health TeleECHO (Extension for Community Healthcare Outcomes) was established at the University of New Mexico Health Sciences Center (UNM HSC) through collaboration of the ECHO Institute and the Osteoporosis Foundation of New Mexico. It is the prototype for technology enabled collaborative learning to expand capacity to deliver best practice skeletal healthcare worldwide. The ECHO model of learning uses videoconferencing to link participants located anywhere there is an electronic connection. Learning is focused on interactive case-based discussions that recapitulate familiar learning strategies of postgraduate medical training programs. Since the launch of the first Bone Health TeleECHO, additional programs have been started in other US states and other countries. This is a report of the progress and challenges in the global development of Bone Health TeleECHO.

The proof-of-concept Bone Health TeleECHO program at UNM HSC was launched on October 5, 2015. Weekly (excluding holidays) videoconferences have been held since that time. Other Bone Health TeleECHO programs are based at locations that include Grand Blanc, Michigan; Washington, DC; Chicago, IL; Galway, Ireland; and Moscow, Russia. More are anticipated. Challenges for initiating and maintaining these include funding, staffing, recruitment of participants, and bureaucratic barriers.

Bone Health TeleECHO uses state-of-the-art communication technologies to connect participants to advance their level of knowledge, with the goal of making them better equipped to manage patients with bone diseases. It offers educational opportunities with minimal disruption to office routines and relieves professional isolation that commonly occurs in a wide range of practice settings. Through replication and innovation in many global locations, Bone Health TeleECHO leverages scarce resources and expands capacity to provide better bone health care for more patients closer to home, with greater convenience and lower cost than referral to a specialty center.

\section{NSS6 \\ NATIONAL UNIVERSITY OF IRELAND GALWAY BONE HEALTH TELEECHO}

J. J. Carey ${ }^{1}$

${ }^{1}$ National University of Ireland Galway, Galway, Ireland

Ireland has a long history of using telemedicine for the care of patients with musculoskeletal diseases. It has been found that patient care delivered remotely with electronic technology is concordant with in-person visits for assessment, diagnosis, and patient satisfaction. Online learning has subsequently been expanded to educate primary care physicians in the care of musculoskeletal diseases. When virtual clinics were included as part of a fracture liaison service (FLS), osteoporosis treatment rates rose to over $80 \%$ for patients admitted to hospital for a hip fracture.

In 2012, staff at National University of Ireland, Galway (NUIG) traveled to the ECHO Institute at University of New Mexico Health Sciences Center in Albuquerque, New Mexico, USA. Intensive on-site training in the ECHO model of learning was provided at no cost in anticipation of launching NUIG Bone Health TeleECHO in 2012. The program was subsequently discontinued due to limited funding and loss of key staff, then reestablished in 2018. The program is now doing well, but challenges remain with limited sources of funding, limited time of participants, uncertain administrative support, and scheduling logistics.

\section{NSS7 \\ BONE HEALTH TELEECHO MOSCOW}

Z. E. Belaya $^{1}$

${ }^{1}$ The National Medical Research Centre for Endocrinology, Moscow, Russia

The ECHO model of learning and care management was developed at the University of New Mexico Health Sciences Center (USM HSC) in 2003. Project ECHO has since expanded to address many diseases and to include other countries. Bone Health TeleECHO was developed at the USM HSC with the aim to improve osteoporosis care and serve as a model for replication in other states and countries. Bone Health Tele ECHO Moscow has replicated the ECHO model to improve osteoporosis care in Russia. Each Russian TeleECHO session includes a short didactic presentation and discussion of clinical cases in a monthly 75-minute videoconference. Since the Russian continuing medical education system does not have the option to provide 
CME credits with teleECHO zoom based programs, all TeleECHO sessions are available as simultaneously recorded webinars.

Over the first 11 months of Bone Health TeleECHO Moscow there were 701 participants with the mean number 64 participants per session from all parts of Russia and other countries (Belarus, Kazakhstan, Armenia). In addition to this 1543 participants watched the simultaneously recorded webinars. Physicians' self-confidence in osteoporosis care was assessed with a questionnaire which incorporated the 20 domains of osteoporosis care. The results showed substantial improvement with ECHO intervention among 131 participants who confirmed that they attended from 3 to 11 Bone Health TeleECHO clinics. The respondents (125 women and 6 men, mean age 41 (23-70), mainly endocrinologists $(n=116)$ and other physicians) represented all the Federal Districts of Russia (19 from Southern; 61 from Central; 11 from Northwestern; 13 from Volga; 11 from Urals; 15 from Siberian and 1 from Eastern Federal Districts). There was a statistically significant $(\mathrm{p}<0.01)$ overall improvement in confidence of care for osteoporosis patients from slight to average or from average to competent with an effect size of 0.75 as classified according to the method of Cohen and Sawilowsky. In conclusion, Bone Health TeleECHO is effectively replicated in Russia with similar self-confidence outcomes, allowing access in local time zone and language.

\section{NSS8}

\section{RARE BONE DISEASE TELEECHO}

K. Javaid ${ }^{1}$

${ }^{1}$ NIHR Musculoskeletal Biomedical Research Unit, University of Oxford, Oxford, United Kingdom

The management of rare bone diseases is challenging due to lack of knowledge for many of them, the scarcity of experienced experts, and often long distances between specialty centers and patients who could benefit from care. To address these unmet needs, Rare Bone Disease TeleECHO was established in 2019. The program is based with the Osteogenesis Imperfecta Foundation in Gaithersburg, Maryland, USA, with collaboration of the Rare Bone Disease Alliance. It is funded by grants through the Osteoporosis Foundation of New Mexico and directed by Laura Tosi, MD. The goal is to expand capacity to safely and effectively diagnose and treat rare bone diseases and disorders worldwide. Healthcare professionals interested in participating can register online at https://www.surveymonkey. com/r/rareboneECHO. Continuing medical education (CME) credits are offered at no charge.

In each monthly one-hour interactive videoconference, faculty members or guest speakers present a brief didactic presentation, followed by participant-led case presentations and discussions. The faculty encourages participants to present case studies related to rare bone diseases at each session. The experience to date has been extremely favorable, with a broad range of specialties participating from locations worldwide. There are typically at least 50 individuals attending each month.

Project ECHO is an innovative educational program developed at the University of New Mexico Health Sciences Center. This collaboration assists in expanding access to specialty care by increasing capabilities of primary care providers, improving the health of the beneficiary population, decreasing costs associated with rare bone disease care, and ensuring a ready medical force.

\section{NSS9 \\ PRINCIPLES OF DEEP LEARNING AND MACHINE LEARNING}

J. A. Carrino

${ }^{1}$ Department of Radiology and Imaging, Hospital for Special Surgery, New-York, United States

Picture archiving and communications systems (PACS) are the information system paradigm used to transmit, store, retrieve and view large amounts of medical imaging data. The transition from the "lightbox paradigm" to the "workstation paradigm" provides an opportunity to electronically explore the significant determinants of image interpretation, develop optimal workflow patterns and create an ideal "person-machine" interface.

Artificial intelligence (AI) algorithms, particularly deep learning, have demonstrated remarkable progress in imagerecognition tasks such as detection, characterization and monitoring of diseases. In addition, AI can impact of the organizational structure on the delivery, quality, cost, and access to health care resources.

AI has recently received attention in medical imaging in part because of substantial improvements in image recognition performance, based largely on a class of algorithms known as deep learning. Deep learning is a class of machine learning algorithms that uses multiple layers to progressively extract higher-level features from the raw input. Machine learning carries the connotation that it is extremely complex. While it is methodologically rigorous it is more simple when expressed in mathematical terms. This presentation will describe the concepts common to all machine learning and provides a description of deep learning methods and components.

\section{NSS10}

ARTIFICIAL INTELLIGENCE IN VARIOUS MODALITIES OF MUSCULOSKELETAL IMAGING (RADIOGRAPHY, DXA, CT, MRI)

R. M. Summers ${ }^{1}$

${ }^{1}$ Diagnostic Radiology Department, Imaging Biomarkers and Computer-Aided Diagnosis (CAD) Laboratory, NIH Clinical Center, Bethesda, United States 
There has been explosive growth recently in research on artificial intelligence in musculoskeletal imaging. This growth is attributable to advances made in machine learning by researchers in the fields of computer vision and medical image analysis. The potential clinical applications of artificial intelligence in musculoskeletal imaging are many, including fullyautomated screening for osteoporosis, fracture detection, bone age assessment, degenerative disease quantification and oncology applications. The potential benefits to the patient are also many, including a reduction in diagnostic errors and interobserver variability, dissemination of expert knowledge through algorithms trained on relevant populations, and timelier image interpretation. This presentation will highlight these applications and benefits, and will attempt to put these novel developments in artificial intelligence in musculoskeletal imaging in perspective.

\section{NSS11}

AUTOMATED KNEE OSTEOARTHRITIS ASSESSMENT BY AI INCREASES PHYSICIANS' AGREEMENT RATE AND ACCURACY

\section{S. Nehrer ${ }^{1}$}

${ }^{1}$ Center for Regenerative Medicine and Center for Health Sciences and Medicine, Medical Faculty, Danube University Krems, Krems, Austria

Objective: The diagnosis of knee osteoarthritis depends on the identification and classification of several radiographic features, such as presence and degree of osteophytes, sclerosis, and joint space narrowing. Here, we assess the impact of a computerized system on physicians' accuracy and agreement rate, as compared to unaided diagnosis.

Methods: A set of 124 unilateral knee radiographs from the OAI study were selected and analyzed by a deep learning-based method with regard to Kellgren-Lawrence (KL) grade, as well as Joint Space Narrowing, Osteophytes and Sclerosis OARSI grades. Physicians were instructed to score all images, with respect to these features, in two modalities: being shown simply the image of a radiograph (unaided) and when presented with the report from the computer assisted detection system (aided). The two reading sessions were separated by an appropriate washout period. The readers were blinded to each other's grades and to the ground truth grading (OAI consensus grades). Agreement rates (Intra-Class Correlation - ICC) between the physicians were calculated for both modalities. Furthermore, the physicians' performance was compared to the ground truth grading (OAI consensus), and accuracy, sensitivity and specificity in both modalities were calculated for each feature.

Results: Agreement rates (ICC) for KL grade, sclerosis, and osteophyte OARSI grades, were statistically increased in the aided modality vs the unaided modality. Readings for Joint
Space Narrowing OARSI grade did not show a statistical difference between the two modalities. Readers' accuracy for detection of any abnormality $(\mathrm{KL}>0)$, osteoarthritis $(\mathrm{KL}>1)$, sclerosis (sclerosis OARSI grade $>0$ ), and osteophytosis (osteophyte OARSI grade $>0$ ) was significantly increased in the aided modality. These increases in accuracy were driven by significant increases in specificity, with no statistical difference in sensitivity.

Conclusions: These results show the use of an automated knee osteoarthritis software increases consistency between physicians when grading radiographic features of OA. Furthermore, the use of a software solution increases specificity with no losses in sensitivity.

\section{NSS12}

\section{CURRENT CHALLENGES FOR PRIMARY CARE} OSTEOPOROSIS MANAGEMENT

\section{Z. Paskins $^{1}$}

${ }^{1}$ Senior Lecturer and Honorary Consultant Rheumatologist, Primary Care Centre Versus Arthritis, Keele University, Keele, United Kingdom

The context for this symposium will be set using findings of a synthesis of qualitative research which provides some insight into the challenges primary care clinicians report in managing people with osteoporosis. Findings from empirical qualitative research which investigated the barriers and facilitators to incorporating fracture risk assessments in integrated primary care reviews will also be presented.

\section{NSS13}

WHAT OSTEOPOROSIS CARE LOOKS LIKE IN CURRENT UK GENERAL PRACTICE

E. Cottrell ${ }^{1}$

${ }^{1}$ General Practitioner and Senior Lecturer in General Practice, Keele University, Keele, United Kingdom

Using existing literature, findings from ongoing studies and real world audit data, the current primary care provision of osteoporosis management will be outlined.

\section{NSS14}

PRIMARY CARE AND THE MANAGEMENT OF SPECIAL GROUPS AT RISK OF FRACTURE

E. Cottrell ${ }^{1}$

${ }^{1}$ General Practitioner and Senior Lecturer in General Practice, Keele University, Keele, United Kingdom

Using real world audit data practical decision making and dilemmas encountered in primary care for special populations (e.g. those with eating disorders, chronic pancreatitis and previous bariatric surgery) will be highlighted. 


\section{NSS15}

\section{HOW PRIMARY CARE OSTEOPOROSIS MANAGEMENT CAN BE IMPROVED}

J. Edwards $^{1,2}$

${ }^{1}$ General Practitioner and a Senior Lecturer in General Practice, Keele University, Keele, United Kingdom, ${ }^{2}$ Member of the UK National Osteoporosis Guidelines Group and the Royal Osteoporosis Society Bone Academy Clinical Effectiveness Working Group, Keele, United Kingdom

A practical approach to the roles of primary care in the management of fracture risk, using the best available evidence and available primary care resource will be outlined. Through a worked example, the means by which quality of primary care for fracture risk management can be measured will be demonstrated.

\section{NSS16}

S U M M I G U P : C HAL L E N E S A N D OPPORTUNITIES FOR BETTER INTEGRATION OF PRIMARY AND SECONDARY CARE

\section{Z. Paskins}

${ }^{1}$ Senior Lecturer and Honorary Consultant Rheumatologist, Primary Care Centre Versus Arthritis, Keele University, Keele, United Kingdom

The implications for clinical practice and future research will be set, incorporating findings from the previous sessions.

\section{NSS17 \\ COLLABORATION FOR BETTER PATIENT OUTCOMES IN VERTEBRAL FRACTURE \\ J. Griffin ${ }^{1}$ \\ ${ }^{1}$ Clinical Lead- Quality improvement, Royal Osteoporosis Society, Bath, United Kingdom}

Vertebral fragility fractures are associated with increased fracture risk, morbidity and mortality and only come to clinical attention in approximately $30 \%$ of cases. A large unmet need to identify those with high fracture risk who have not previously had vertebral fractures recognised is well understood and the Royal Osteoporosis Society (ROS) places high importance on this activity.

In 2017 the ROS published a clinical guidance to support the identification of vertebral fragility fractures within imaging departments and direct people with these fracture to appropriate secondary fracture prevention services.

The guidance served also to inspire the Royal College of Radiologists (RCR) audit programme to collaborate with the ROS in developing a national audit on the radiological reporting practice of vertebral fragility fractures as incidental findings on computed tomography (CT) scans in the UK.
Working together with the support from the Royal College of Physicians Falls and Fragility Fracture Audit Programme the ROS and RCR developed audit questions to evaluate adherence to the ROS standards set out in the guidance.

The questions also interrogated the use of outsourcing reporting, infrastructure and clinical pathways.

This unique collaboration also paved the way for RCR members to access quality improvement support and advice from the ROS and make links between radiology and secondary fracture prevention services which rarely interact.

NSS18

NATIONAL AUDIT ON THE REPORTING OF I N C I D E N T A L L Y FO UND VERTEBRAL FRACTURES IN ROUTINE RADIOLOGY PRACTICE N. Mahmood ${ }^{1}$

${ }^{1}$ Royal College of Radiologists, London, United Kingdom

Objectives: To evaluate organisational reporting infrastructure and patient-related reporting data in the diagnosis of vertebral fragility fractures (VFF's) as demonstrated on computed tomography (CT).

Methods: Ethical approval for this type of study is not required in the UK. All UK radiology departments with an audit lead registered with The Royal College of Radiologists (RCR) were invited to participate in this retrospective audit. Organisational and patient specific questionnaires were completed with $\mathrm{CT}$ reporting data acquired from 50 consecutive non-traumatic studies including the thoracolumbar spine.

Results: 127 out of 202 departments (63\%) supplied data to the study, with inclusion of 6357 patients. There was a lack of compliance with all audit standards: $79 \%$ of reports commented on bone integrity (target 100\%), fracture severity was mentioned in $26.2 \%$ (target $100 \%$ ), the recommended terminology 'vertebral fracture' was used in $60.3 \%$ (target $100 \%$ ) and appropriate onward referral was recommended in $2.6 \%$ (target 100\%).

Conclusions: The findings from this study should be used to improve the diagnosis and care for patients with osteoporotic VFF's. Solutions are multifactorial, but radiologist and FLS engagement is fundamental. This audit forms the baseline for further evaluation and a springboard for quality improvement work in the field, with RCR faculty, members and beyond.

\section{NSS19}

EVALUATION OF THE IDENTIFICATION OF VERTEBRAL FRACTURES WITHIN FRACTURE LIAISON SERVICES- LOOKING TO THE FUTURE M. K. Javaid ${ }^{1}$

${ }^{1}$ Nuffield Department of Orthopaedics, Rheumatology and Musculoskeletal Sciences, University of Oxford, Oxford, United Kingdom 
The RCR audit has provided a foundation for relationships between Radiology and FLS and a baseline for further quality improvement work, around VFFs, across Radiology, the ROS and within the Royal College of Physicians Falls and Fragility Fracture Audit Programme (RCP FFFAP). The FFFAP manages the UK wide Fracture Liaison Service Database (FLSDB)an audit of 71 FLS' practice against key performance indicators evidencing efficacy of these services with over 220,000 patient records to date.

In 2020 FLSDB is auditing the ability, effectiveness and resource required for FLS' to identify VFFs though radiology reporting. From a preparatory audit, $68 \%$ of FLS' planned to use CT and $60 \%$ MR to identify vertebral fractures however less than $60 \%$ would report their expected weekly number of scans. 26\% FLS' felt unable to search radiology reports for specific phrases and 8 separate radiology reporting systems were in use across all the FLS'. 65\% of FLS' would need training on VF identification and only $50 \%$ had identified a local radiologist.

The findings will be used to run a pilot of the main audit in January 2020 and then run the main RCP audit in September 2020. The finding of the audit will contextualise the effect of RCR reporting practice in clinical practice and builds an evidence base for the development of resourcing tools to support business case development for FLS'.

\section{NSS20}

EVALUATING THE DEMAND ON FRACTURE LIAISON SERVICES WITH EFFECTIVE IDENTIFICATION OF VERTEBRAL FRACTURES

H. Glenn $^{1}$

${ }^{1}$ Royal Osteoporosis Society, Bath, United Kingdom

The ROS clinical guidance and RCR audit has raised the profile of identifying vertebral fragility fractures (VFFs) through routine clinical practice in the UK. This additional scrutiny has driven the need to understand the additional demand for FLS capacity where effective systems are in place to identify VFFs.

To address this the ROS has developed a demand model.

The demand model is informed by radiology audit data inputted at a local level based on the centre's demographics and specialisms. The model then proportions data depending on specific clinical criteria that maps to the ROS decision tree for VFFs referral to FLS. The final output being the number of new FLS referrals per number of radiology examinations routinely undertaken in a given period of time. E.g. per month.

This estimated demand is used to support services in understanding their local demand and to inform FLS business cases for resource to manage that demand.

\section{NSS21}

\section{INTRODUCTIONS AND WELCOME/AIMS AND METHODS}

E. Harding ${ }^{1}$

${ }^{1}$ The Health Policy Partnership (HPP), London, United Kingdom

The lecturer will provide background information on Osteoporosis and fragility fractures: a policy toolkit - a comprehensive resource for European policymakers which was launched online in January 2020. The policy toolkit was developed based on a pragmatic, evidence-focused literature review and expert interviews. Feedback and input was provided by an informal multidisciplinary group of expert stakeholders working at European and national level, including a Parliamentary Forum comprising former parliamentarians. The toolkit includes a call to action with specific asks for policymakers working at the European, national and regional levels. It has been endorsed by pan-European and national organisations. In addition, it includes ten country profiles (Belgium, Finland, France, Germany, Ireland, Italy, the Netherlands, Romania, Spain, the UK).

This work was initiated and funded by Amgen (Europe) $\mathrm{GmbH}$. The initial phase of work seeks to analyse and raise awareness of health system barriers to clinical best practice in osteoporosis care and fracture prevention in the EU. All outputs are non-promotional and based on existing literature and expert stakeholder consensus. Amgen (Europe) GmbH did not have editorial control.

\section{NSS22}

\section{CRITICAL CHALLENGES IN OSTEOPOROSIS AND} FRAGILITY FRACTURE CARE

C. Cooper $^{1,2,3,4}$

${ }^{1}$ President, International Osteoporosis Foundation, Nyon, Switzerland, ${ }^{2}$ Professor of Rheumatology and Director, MRC Lifecourse Epidemiology Unit, Southampton, United Kingdom, ${ }^{3}$ Vice-Dean of Medicine, University of Southampton, Southampton, United Kingdom, ${ }^{4}$ Professor of Epidemiology, Nuffield Department of Orthopaedics, Rheumatology and Musculoskeletal Sciences, University of Oxford, Oxford, United Kingdom

The lecturer will summarise key challenges in osteoporosis and fragility fracture care across Europe. This includes an absence of clear guidance for screening; gaps in early diagnosis and treatment of osteoporosis in primary care and postfracture; a general lack of awareness and knowledge about osteoporosis and fragility fractures and low prioritisation at policy level, often leading to restricted reimbursement structures and little attention at care delivery level.

This work was initiated and funded by Amgen (Europe) $\mathrm{GmbH}$. The initial phase of work seeks to analyse and raise 
awareness of health system barriers to clinical best practice in osteoporosis care and fracture prevention in the EU. All outputs are non-promotional and based on existing literature and expert stakeholder consensus. Amgen (Europe) GmbH did not have editorial control.

\section{NSS23}

POLICY ACTIONS NEEDED TO IMPROVE PREVENTION AND CARE OF OSTEOPOROSIS AND FRAGILITY FRACTURES

\section{J. Bowis ${ }^{1,2,3}$}

${ }^{1}$ Honorary Patron, Health First Europe, Brussels, Belgium,

${ }^{2}$ Former Health Minister, London, United Kingdom,

${ }^{3}$ Member of the European Parliament for the UK, Brussels,

Belgium

The lecturer will present the core policy 'asks' contained in the toolkit. These are directed to European, national, and regional-level policymakers. The lecturer will also present five building blocks identified in the toolkit as essential for an effective policy response. These include: 1. Building a system that works: policies for scrutiny, accountability and investment, 2. Catching it early: detection and management in primary care, 3 . Getting people back on track: facilitating multidisciplinary care post-fracture, 4 . Supporting quality of life as part of healthy and active ageing: prevention of falls and fractures in later life, 5. Engaging patients and public: Awareness, activation and self-management.

This work was initiated and funded by Amgen (Europe) $\mathrm{GmbH}$. The initial phase of work seeks to analyse and raise awareness of health system barriers to clinical best practice in osteoporosis care and fracture prevention in the EU. All outputs are non-promotional and based on existing literature and expert stakeholder consensus. Amgen (Europe) GmbH did not have editorial control.

\section{NSS24}

RANKL INHIBITION IMPROVES MUSCLE STRENGTH AND INSULIN SENSITIVITY AND RESTORES BONE MASS FROM MICE TO HUMANS N. Bonnet ${ }^{1}$, L. Bourgoin ${ }^{1}$, E. Biver ${ }^{1}$, E. Douni ${ }^{2}$, S. Ferrari ${ }^{1}$ ${ }^{1}$ Division of Bone Diseases, Geneva University Hospitals and Faculty of Medicine, Geneva, Switzerland, ${ }^{2}$ Biomedical Sciences Research Center Alexander Fleming, Athens, Greece

Receptor activator of NFKB ligand (RANKL) activates, while osteoprotegerin (OPG) inhibits, osteoclastogenesis. In turn a neutralizing $\mathrm{Ab}$ against RANKL, denosumab, improves bone strength in osteoporosis. OPG has also been shown to improve muscle strength in mouse models of Duchenne's muscular dystrophy $(\mathrm{mdx}$ ) and denervation-induced atrophy, but its role and mechanisms of action on muscle weakness in other conditions remains to be investigated. We investigated the effects of RANKL inhibitors on muscle in osteoporotic women and mice that either overexpress RANKL (HuRANKL$\mathrm{Tg}+$ ) or lack Pparb and concomitantly develop sarcopenia (Pparb-/-). In women, denosumab over 3 years improved appendicular lean mass and handgrip strength compared to no treatment, whereas bisphosphonate did not. HuRANKL-Tg+ mice display lower limb force and maximal speed, while their leg muscle mass is diminished, with a lower number of type I and II fibers. Both OPG and denosumab increase limb force proportionally to the increase in muscle mass. They markedly improve muscle insulin sensitivity and glucose uptake, and decrease anti-myogenic and inflammatory gene expression in muscle, such as myostatin and protein tyrosine phosphatase receptor- $\gamma$. Similarly, in Pparb-/-, OPG increases muscle volume and force, while also normalizing their insulin signaling and higher expression of inflammatory genes in skeletal muscle. In conclusions, RANKL deteriorates, while its inhibitors improve, muscle strength and insulin sensitivity in osteoporotic mice and humans. Hence denosumab could represent a novel therapeutic approach for sarcopenia.

\section{NSS25}

OLD AND NEW ROLES OF AMINO ACIDS AND AMINO BUTYRIC ACIDS IN MUSCULOSKELETAL DISEASES

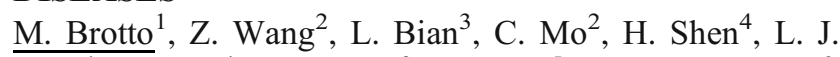

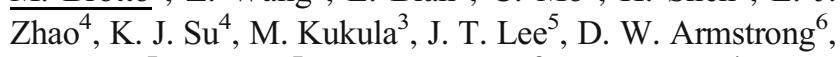
R. Recker ${ }^{7}$, J. Lappe ${ }^{7}$, L. F. Bonewald ${ }^{8}$, H. W. Deng ${ }^{4}$

${ }^{1}$ University of Texas-Arlington (UTA), College of Nursing \& Health Innovation, Bone-Muscle Research Center, Arlington, United States, ${ }^{2}$ Bone-Muscle Research Center, College of Nursing and Health Innovation, University of TexasArlington, Arlington, United States, ${ }^{3}$ Shimadzu Center for Advanced Analytical Chemistry, University of Texas at Arlington, Arlington, United States, ${ }^{4}$ Tulane Center of Bioinformatics and Genomics, Department of Biostatistics and Data Science, Tulane University, New Orleans, United States, ${ }^{5}$ AZYP LLC - Separation \& Analytics, Arlington, United States, ${ }^{6}$ Department of Chemistry and Biochemistry, College of Science, University of Texas at Arlington, Arlington, United States, ${ }^{7}$ School of Medicine Osteoporosis Research Center, Creighton University, Omaha, United States, ${ }^{8}$ Indiana Center for Musculoskeletal Health, School of Medicine, Indiana University, Indianapolis, United States

The World Health Organization Burden of Disease Report informed that musculoskeletal diseases afflict $1 / 3$ of the world's population with tremendous burden on societies and individuals. Osteosarcopenia (defined as combined osteoporosis and sarcopenia) affects older adults around the world and many times has devastating complications that affect their well-being and quality of life. During the last decade, we 
proposed to look at bone and muscle from a different view, particularly looking at its interactions. We referred to this phenomenon as bone-muscle crosstalk. Others and we discovered that bone and muscles secrete a number of molecules (myokines and osteokines) that are necessary for reciprocal optimal function. We now also have new evidence showing that amino acids, their metabolites, and aminobutyric acids may play an important role in bone-muscle crosstalk. Furthermore, we started exploring the potential utilization of some of these molecules for the diagnosis of musculoskeletal diseases. Specifically, we have developed a new metabolomics approaches to quantify these molecules. We recently applied these metabolomics approaches along with RNAseq and Bioinformatics to investigate their associations with bone mineral density in young and older women and in women with and without bone fractures. We will present and discuss these exciting new findings and propose the utilization of aminobutyric acids as a new class of molecules that holds great potential to improve early diagnosis and treatments of musculoskeletal diseases, particularly osteosarcopenia.

\section{NSS26}

\section{FAT AS A THERAPEUTIC TARGET IN OSTEOSARCOPENIA}

G. Duque $^{1,2}$, B. Kirk ${ }^{1,2}$, A. Al Saedi ${ }^{1,2}$

${ }^{1}$ Australian Institute for Musculoskeletal Science, The University of Melbourne and Western Health, Melbourne, Australia, ${ }^{2}$ Department of Medicine-Western Health, Melbourne Medical School, The University of Melbourne, Melbourne, Australia

Fat infiltration is one of the hallmarks of sarcopenia and osteoporosis, thus of osteosarcopenia. High levels of marrow adipose tissue (MAT) are associated with bone loss and osteoporosis. MAT secretes adipocytokines and free fatty acids (FFAs), which are toxic to the cells in the vicinity of adipocytes, decreasing bone formation and increasing bone resorption. Similarly, fat infiltration in muscle fibers is associated with cell dysfunction. Amongst those MAT-secreted factors, FFAs (and predominantly palmitic acid), have demonstrated the stronger toxic effect on bone and muscle cells thus allowing us to propose that inhibition of fatty acid synthase in MAT could have an anabolic effect on muscle and bone. Our previous evidence in oophorectomized (OVX) mice has confirmed this hypothesis ${ }^{1}$. Treatment with an inhibitor of fatty acid synthase attenuated bone loss in OVX mice. In addition, cerulenin-treated OVX mice showed an increase in muscle mass and function. Overall, this preliminary evidence suggests that MAT-derived products could become a novel therapeutic target with dual anabolic effect on muscle and bone. A hypothesis that deserves further exploration.

${ }^{1}$ Bermeo S, Al Saedi A, Vidal C, Khalil M, Pang M, Troen BR, Myers D, Duque G. Treatment with an inhibitor of fatty acid synthase attenuates bone loss in ovariectomized mice. Bone. 2019 May;122:114-122

NSS27

DIVERSITY OF THE PATHOPHYSIOLOGY OF INCREASED BONE FRAGILITY IN RARE BONE DISEASES

N. A. T. Hamdy ${ }^{1}$

${ }^{1}$ Department of Medicine, Division of Endocrinology \& Center for Bone Quality, Leiden University Medical Center, Leiden, The Netherlands

Objective: To increase awareness for the diverse pathophysiological mechanisms contributing to increased bone fragility in different rare bone diseases.

Methods: Overview of the different factors contributing to poor bone quality, decreased bone strength and increased bone fragility in various rare bone diseases.

Results: Determinants of bone strength include the structural (bone microarchitecture) and material (collagen and mineral) properties of bone, also determined by the cellular activities of bone modeling and remodeling, the disturbance of any of these components, potentially contributing to bone fragility, and increased fracture risk, in addition or independently of changes in bone mineral density. Insight is provided into the diversity of factors which may variably contribute to increased bone fragility by disrupting bone microarchitecture and geometry, altering the composition of bone tissue, altering bone remodeling and altering bone mineralization. Examples used to illustrate the contribution of these diverse mechanisms to increased fracture risk in rare bone disorders are the abnormal or decreased synthesis of type I collagen in osteogenesis imperfecta, the abnormal mineralization due to phosphate wasting in X-linked hypophosphatemic rickets (XLH), the failure of osteoclast development or function in osteopetrosis or the deficient activity of cathepsin $\mathrm{K}$ in pyknodysostosis, despite increased bone density in both disorders, the monostotic or polyostotic focal abnormal bone formation and mineralization in fibrous dysplasia/McCune Albright syndrome, and the mineralization defect associated with loss of function mutation of the tissue nonspecific isoenzyme of alkaline phosphatase (TNSALP) gene in hypophosphatasia.

Conclusion: Unravelling the diverse cellular and molecular pathophysiology of these rare bone diseases has paved the way for the development of therapies specifically targeting the abnormalities responsible for the pathophysiology of at least two of these disorders: the monoclonal anti-FGF23 antibody burosumab in XLH, and the recombinant TNSALP enzyme replacement therapy asfotase alfa in pediatric-onset hypophosphatasia. 


\section{NSS28}

\section{BONE QUALITY IN OSTEOGENESIS IMPERFECTA} AND EFFECTS ON BONE FRAGILITY LUCA SANGIORGI

L. Sangiorgi $^{1}$

${ }^{1}$ Department of Medical Genetics and Rare Orthopaedic Diseases \& CLIBI Laboratory, IRCCS Istituto Ortopedico Rizzoli, Bologna, Italy

Objective: to describe briefly the bone properties in Osteogenesis Imperfecta (OI) and its relationship with bone fragility observed in the disease.

Methods: Overview about bone quality, architecture and fragility in OI and related pathogenic mechanisms.

Results: Bone fragility leading to recurrent fractures is the main clinical feature in OI, for this reason it was also named "brittle bone disease". A number of factors contribute to this features.

Most cases of OI are related to mutations in COL1A1 and COL1A2 genes, causing abnormalities in collagen molecules and fibers. In OI bone we observe an abnormal bone matrix with hypermineralization. The brittleness is related to both bone matrix and architectural abnormalities. The lower trabecular number and thinner cortices, increase cortical porosity and the fracture risk, due to a reduction of the capacity of energy dissipation in bone for a modification of the mechanical properties of the OI bone.

OI type V and VI represent particular cases. In OI type V, histological examination shows bone lamellation is coarse or mesh-like and no alteration in mineralization rate. In OI type VI hystological appearance is typical fish-scale pattern of bone lamellation under polarized light, unmineralized osteoid, primary mineralization defect.

To monitor therapy effects and bone density, X-ray absorptiometry (DXA) is used, but it doesn't give detailed information about bone architecture. To quantify the geometric, microstructural, densitometric, and mechanical properties of human cortical and trabecular bone other methods should be applied, but they are not employed in standard clinical practice, as we can see in the few studies performed to study the microstructural properties of bone.

Conclusion: in OI lower trabecular number and connectivity as well as lower trabecular thickness and volumetric bone mass contribute to bone fragility; methods to study bone architecture could help to understand mechanisms of bone fragility and have new parameters related to fractures risk.

\section{Reference}

1. Bishop N. Bone Material Properties in Osteogenesis Imperfecta J Bone Miner Res. 2016 Apr;31(4):699708. doi: 10.1002/jbmr.2835. Epub 2016 Mar 26. Review

2. Abidin AZ, Jameson J, Molthen R, Wismüller A. Classification of micro-CT images using 3D characterization of bone canal patterns in human osteogenesis imperfecta. Proc SPIE Int Soc Opt Eng. 2017 Mar;10134. pii: 1013413. doi: 10.1117/ 12.2254421 .

3. Mikolajewicz N, Bishop N, Burghardt AJ, Folkestad L, Hall A, Kozloff KM, Lukey PT, Molloy-Bland M, Morin SN, Offiah AC, Shapiro J, van Rietbergen B, Wager K, Willie BM, Komarova SV, Glorieux FH HR-pQCT Measures of Bone Microarchitecture Predict Fracture: Systematic Review and Meta-Analysis. J Bone Miner Res. 2019 Oct 23. doi: 10.1002/jbmr.3901.

4. Fratzl-Zelman N, Misof BM, Klaushofer K, Roschger P. Bone mass and mineralization in osteogenesis imperfecta Wien Med Wochenschr. 2015 Jul;165(1314):271-7. doi: 10.1007/s10354-015-0369-2. Epub 2015 Jul 25 .

\section{NSS29}

\section{VITAMIN D DEFICIENCY AND AUTOIMMUNITY}

I. Kostoglou-Athanassiou ${ }^{1}$

${ }^{1}$ Department of Endocrinology, Asclepeion Hospital, Voula, Athens, Greece

Vitamin D is a hormone involved in the regulation of musculoskeletal health. It regulates intestinal calcium absorption and it is involved in muscular integrity. Vitamin D also has extraskeletal functions which are in the center of research activities. One of the main extraskeletal functions of vitamin $\mathrm{D}$ is the regulation of the immune system.

Various cells of the immune system express the vitamin D receptor (VDR) and harbor $1 \alpha$-hydroxylase and are therefore capable of locally producing active $1,25(\mathrm{OH})_{2} \mathrm{D}_{3}$. Thus, cells of the immune system respond to vitamin $\mathrm{D}$ and activate vitamin D in a paracrine or autocrine fashion. Cells of the immune system which express the VDR and harbor $1 \alpha$ hydroxylase include macrophages, T cells, dendritic cells, monocytes and B cells. Vitamin D is involved in the regulation of the innate immunity as it enhances the defense system of the organism against microbes and other pathogenic organisms and it modulates the adaptive immune system through direct effects on $\mathrm{T}$ cell activation and on the phenotype and function of antigen-presenting cells particularly dendritic cells. $1,25(\mathrm{OH})_{2} \mathrm{D}_{3}$ suppresses $\mathrm{T}$ cell receptor induced $\mathrm{T}$ cell proliferation and changes cytokine expression. The overall shift is away from $\mathrm{T}$ helper Th1 phenotype toward a more tolerogenic Th2 response. Additionally, $1,25(\mathrm{OH})_{2} \mathrm{D}_{3}$ suppresses autoimmunity and tissue destruction by inhibiting the Th17 response at several levels. Vitamin D also appears to have a direct effect on B cells by inhibiting immunoglobulin production and interrupting $\mathrm{B}$ cell differentiation. $1,25(\mathrm{OH})_{2} \mathrm{D}_{3}$ has effects on dendritic cells. Physiologic levels of $1,25(\mathrm{OH})_{2} \mathrm{D}_{3}$ inhibit maturation of dendritic cells and maintain an immature and tolerogenic phenotype with inhibition of activation markers and up-regulation of inhibitory molecules. 
Vitamin D is a potent immunomodulator and it has multiple and diverse effects on the immune system. It appears that the vitamin D status of an individual may have important immunologic consequences. In animal models of autoimmunity, it may prevent or ameliorate the expression of autoimmune disease. In the human, autoimmune diseases may be prevented or their clinical profile may be modulated by vitamin D.

\section{NSS30}

\section{VITAMIN D DEFICIENCY AND RHEUMATOID} ARTHRITIS

Y. Dionyssiotis $^{1}$

${ }^{1}$ 1st Physical Medicine \& Rehabilitation Department, National Rehabilitation Center EKA-KAT, Athens, Greece

Vitamin D is vital for calcium homeostasis and it is involved in the regulation of the immune system. Deficiency of vitamin $\mathrm{D}$ is associated with autoimmune diseases. Vitamin D deficiency is associated with an elevated risk for rheumatoid arthritis (RA).

A meta-analysis showed that low vitamin D intake is associated with the development of RA. Thereafter, several studies performed in various areas all over the world showed that vitamin D deficiency is observed in patients with RA and that vitamin $\mathrm{D}$ deficiency is associated with disease activity. A meta-analysis of the good quality studies performed regarding the association between vitamin D deficiency and RA showed that vitamin D deficiency is observed in RA patients significantly more than in a control group and that vitamin D levels are inversely correlated with disease activity, meaning that low vitamin $\mathrm{D}$ levels are associated with high disease activity. Moreover, RA incidence is increased in vitamin D deficient subjects and an inverse relationship exists between serum vitamin D levels and RA activity.

More studies are needed to explore the exact association between vitamin $\mathrm{D}$ deficiency and RA and to determine the best method of vitamin D supplementation and whether it may be used for the prevention of RA or for the best management of the disease. It has been proposed that vitamin D may contribute to the management of pain in RA and may be used supplementary to TNF- $\alpha$ inhibitors in RA treatment.

\section{NSS31}

\section{VITAMIN D DEFICIENCY AND SYSTEMIC LUPUS ERYTHEMATOSUS}

P. Athanassiou ${ }^{1}$

${ }^{1}$ Department of Rheumatology, St. Paul's Hospital, Thessaloniki, Greece

Vitamin D has emerged as a potent immunomodulator. It may induce tolerance to self-antigens. On the contrary, vitamin D deficiency may induce loss of tolerance to self-antigens leading thereafter to the development of clinical autoimmune disease. Systemic lupus erythematosus (SLE) is a systemic autoimmune disease involving multiple systems and organs. Vitamin D deficiency has been observed in SLE patients and it may be implicated in disease pathogenesis.

In SLE the inflammatory milieu drives the development of T cells into proinflammatory pathways, defective function of Tregs and survival and activation of $\mathrm{B}$ cells which produce autoantibodies. Patients with SLE have lower $25(\mathrm{OH}) \mathrm{D}_{3}$ levels compared to controls, suggesting that vitamin D deficiency may be a risk factor for SLE. Most of the studies have also found higher SLE disease activity associated with lower levels of $25(\mathrm{OH}) \mathrm{D}_{3}$. As patients with SLE often have photosensitivity and are advised to avoid direct sun exposure, detecting vitamin D deficiency and replacing $25(\mathrm{OH}) \mathrm{D}_{3}$ with oral supplementation is critical and may have an impact on disease activity. It appears that vitamin D deficiency may be observed in patients with SLE, a systemic autoimmune disease and it may be related to disease severity. Vitamin D supplementation along with standard immunomodulatory treatment may be critical for the long-term management of the disease in SLE patients.

\section{NSS32}

\section{VITAMIN D RECEPTOR POLYMORPHISMS AND} AUTOIMMUNE DISEASES

L. Athanassiou $^{1}$

${ }^{1}$ Department of Physiology, Medical School, University of Athens, Athens, Greece

The vitamin D receptor (VDR) is present in the T lymphocyte population increasing in the presence of $1,25(\mathrm{OH})_{2} \mathrm{D}_{3}$. Thus, VDR seems to be implicated in the immune regulating action of vitamin D. VDR polymorphisms may modulate the action of vitamin $\mathrm{D}$ and its immunoregulatory activity.

VDR is the intracellular receptor of vitamin D. It is encoded in chromosome 12 and it consists of 427 amino acids. VDR is implicated in vitamin D action and may be implicated in the immunoregulatory action of the hormone. In animals, in which the VDR was inactivated increased activity of the immune system was observed, mainly due to modulation of regulatory $\mathrm{T}$ cells. More than 60 single nucleotide polymorphisms of the VDR have been observed. FokI, BsmI, ApaI and TaqI have been more extensively studied. FokI polymorphism alters the structure of the receptor. The presence of TaqI increases the risk for the development of rheumatoid arthritis (RA). The presence of FokI increases the risk for RA. VDR polymorphisms have been studied in the context of systemic lupus erythematosus (SLE). TaqI and ApaI polymorphisms have not been found to be associated with the development of SLE. The FokI polymorphism has not been shown to be associated with SLE in Caucasians and Asians, having, however, been found to have such an association in the Arabic population. The expression of Fokl has been shown to be related to the development of lupus and lupus nephritis in the Egyptian population. VDR polymorphisms have been studied in 
the context of multiple sclerosis. FokI, BsmI, ApaI and TaqI have been investigated. The presence of TaqI has been shown to be related to increased risk for the development of multiple sclerosis, while the presence of $B s m \mathrm{I}$ has been found to be associated with multiple sclerosis in the Asian population.

It appears that vitamin $\mathrm{D}$ receptor polymorphisms may modulate vitamin D action. In particular, VDR polymorphisms may alter its immunoregulatory action, thus modulating the expression of autoimmune diseases.

\section{NSS33}

\section{EVIDENCE-CARE GAP IN OSTEOPOROSIS AND OSTEOARTHRITIS}

J. Edwards ${ }^{1,2}$

${ }^{1}$ General Practitioner and a Senior Lecturer in General Practice, Keele University, Keele, United Kingdom, ${ }^{2}$ Member of the UK National Osteoporosis Guidelines Group and the Royal Osteoporosis Society Bone Academy Clinical Effectiveness Working Group, Keele, United Kingdom

An overview of published and clinical experience of gaps between evidence and care resulting from inadequate, misaligned and/or insufficient communication will be provided. These include low levels of uptake of and persistence with bisphosphonate therapy in osteoporosis, as well as low levels of recommendation and uptake of the core treatments (information provision, exercise and weight loss, when needed) for osteoarthritis.

\section{NSS34}

\section{WHAT DO WE ALREADY KNOW ABOUT HOW TO} FRAME EXPLANATIONS?

Z. Paskins $^{1}$

${ }^{1}$ Senior Lecturer and Honorary Consultant Rheumatologist, Primary Care Centre Versus Arthritis, Keele University, Keele, United Kingdom

In this session, the Necessity Concerns Framework will be introduced as a simple psychological theory that helps us understand how patients make decisions about medicines and therefore helps frame explanations to support informed adherence.

\section{NSS35}

TARGETING COMMUNICATION IN THE CLINICIAN-PATIENT CONSULTATION TO IMPROVE UPTAKE OF FRACTURE PREVENTION TREATMENTS: EARLY FINDINGS FROM THE IFRAP STUDY

\section{Z. Paskins ${ }^{1}$}

${ }^{1}$ Senior Lecturer and Honorary Consultant Rheumatologist, Primary Care Centre Versus Arthritis, Keele University, Keele, United Kingdom
$\mathrm{iFraP}$ is a 5-year study to design and test an intervention including a computerised decision aid, to use in the consultation, to promote informed decision making and informed adherence of osteoporosis drugs. Early findings from the iFraP study will be presented including an evidence synthesis of existing patient information resources, and views of stakeholders. These early findings provide a foundation of how to talk about osteoporosis, fracture risk and need for drugs, while addressing patient concerns.

\section{NSS36 \\ WHAT PATIENTS WITH OSTEOARTHRITIS WANT AND NEED TO KNOW TO SUPPORT THEM TO UNDERTAKE SELF-MANAGEMENT: EARLY FINDINGS FROM THE PEP-OA STUDY}

\section{E. Cottrell ${ }^{1}$}

${ }^{1}$ General Practitioner and Senior Lecturer in General Practice, Keele University, Keele, United Kingdom

First outlining existing knowledge about patient interpretation of words and current explanations of osteoarthritis, this lecture will develops previous understanding by focusses on the need to deliver osteoarthritis explanations with a purpose. That is to support both understanding of the condition but to do so in such a way that it prompts understanding of the mechanism of benefit and value of self-management (undertake exercise and weight loss, when necessary). Evidence from stakeholder and patient engagement in an empirical study (PEP-OA) designed to develop an osteoarthritis explanation that is relevant to all affected patients. We will present our learning about how words and concepts are interpret and what is acceptable and appropriate within an osteoarthritis explanation.

\section{NSS37}

\section{LESSONS FOR CLINICAL PRACTICE AND RESEARCH}

J. Edwards ${ }^{1,2}$

${ }^{1}$ General Practitioner and a Senior Lecturer in General Practice, Keele University, Keele, United Kingdom, ${ }^{2}$ Member of the UK National Osteoporosis Guidelines Group and the Royal Osteoporosis Society Bone Academy Clinical Effectiveness Working Group, Keele, United Kingdom

This section will review the evidence for the importance of considering behaviour change principals when undertaking clinical and academic activities relating to explanations of osteoporosis and osteoarthritis in order to increase uptake of and adherence to best evidence treatments. 


\section{NSS38}

PRESENTATION OF THE GETTING TO GOLD PROGRAMME FRAMEWORK AND MILESTONES

K. Javaid ${ }^{1}$

${ }^{1}$ NIHR Musculoskeletal Biomedical Research Unit, University of Oxford, Oxford, United Kingdom

During this presentation we will review the key elements of the programme including the learning objectives, learning design and delivery.

\section{NSS39}

EVALUATION OF THE MENTORSHIP PROGRAMME M. Calo $^{1}$

${ }^{1}$ LATAM Region at International Osteoporosis Foundation, Buenos Aires, Argentina

The Getting to Gold Mentorship programme was the first of its kind and required close evaluation. During this presentation, we will review the methods, results and conclusions from running the programme in Mexico.

\section{NSS40}

\section{EVALUATION OF THE MENTORSHIP PROGRAMME- MENTEES PERSPECTIVE}

\section{A. Olascoaga $^{1}$}

${ }^{1}$ Instituto Nacional de Rehabilitación, Mexico City, Mexico

The Getting to Gold Mentorship programme was the first of its kind and during this presentation, a mentee will review the strengths, weaknesses, threats and opportunities of the programme as well as following results after the first phase of the programme.

\section{NSS41}

\section{NEXT STEPS FOR GETTING TO GOLD}

K. Javaid $^{1}$

${ }^{1}$ NIHR Musculoskeletal Biomedical Research Unit, University of Oxford, Oxford, United Kingdom

During this presentation we will review future plans for the globalisation of the programme at the policy, mentor and scalability perspectives.

\section{NSS42}

THE BURDEN OF OSTEOPOROSIS AND FRAGILITY FRACTURES IN ASIA PACIFIC

\section{J. K. Lee ${ }^{1,2}$}

${ }^{1}$ Deputy Medical Director, Head of Department of Orthopedic Surgery and Consultant Orthopedic Surgeon, Beacon International Specialist Centre, Petaling Jaya, Malaysia, ${ }^{2} \mathrm{Co}-$ Chair, Asia Pacific Fragility Fracture Alliance (APFFA), Petaling Jaya, Malaysia
Of the global population of 7.6 billion people, 4.5 billion live in Asia, representing $60 \%$ of the world's inhabitants. As compared with the beginning of the century, the number of people aged $\geq 65$ years in Asia will more than quadruple by 2050 and increase by almost six-fold by 2100 .

This presentation will review the ongoing major shifts in the demography of the Asia Pacific region and summarise the epidemiology of osteoporosis and fragility fracture in countries across the region. This will include findings from the 2018 Asian Federation of Osteoporosis Societies (AFOS) study which updated hip fracture projections in Asia to midcentury, and recent estimates and future projections included in Asia Pacific regional audits undertaken by the International Osteoporosis Foundation.

The WHO has designated 2020-2030 the Decade of Healthy Ageing. A significant component of our response to the longevity miracle must be to ensure that our older people remain mobile and independent throughout old age. This presentation will highlight the need for major coordinated efforts to improve the quality of care provided to people who are living with osteoporosis, such as the Asia Pacific Consortium on Osteoporosis and Asia Pacific Fragility Fracture Alliance, descriptions of which will follow in this symposium.

\section{NSS43}

ASIA PACIFIC CONSORTIUM ON OSTEOPOROSIS: DEVELOPMENT OF THE FRAMEWORK M. Chandran ${ }^{1,2}$

${ }^{1}$ Senior Consultant and Director, Osteoporosis and Bone Metabolism Unit, Department of Endocrinology, Singapore General Hospital, Singapore, Singapore, ${ }^{2}$ Chairperson, Asia Pacific Consortium on Osteoporosis, Singapore, Singapore

The Asia Pacific (AP) region is comprised of 71 countries with vastly different healthcare systems. By 2050 it is predicted that more than half the world's hip fractures will occur in this region. The Asia Pacific Consortium on Osteoporosis (APCO) was set up in May 2019 with the vision of reducing the burden of osteoporosis and fragility fractures in the Asia Pacific region. It brings together clinical experts from countries and regions across AP to develop solutions to challenges facing osteoporosis management and fracture prevention in this highly populous region of the world. APCO currently has 39 experts from 19 countries in the region. APCO aims to achieve this vision by engaging with relevant stakeholders including health care providers, policy makers and the public to help develop and implement country and region-specific programs for research and the prevention and treatment of osteoporosis and its complication of fragility fractures in the Asia Pacific. The initial APCO project is to implement a Framework for the development of Pan-Asia Pacific 
minimum clinical standards for the screening, diagnosis and management of osteoporosis. A comparative analysis will be conducted on osteoporosis and fracture prevention guidelines, published in the last five years in this region. A template for comparison of guidelines will be based on a modified 5IQ approach, which will evaluate recommendations made concerning patient identification, investigations, information, initiation of pharmacological treatment, integration with primary care and quality measures of the service provided. Informed by the analysis, a Delphi process will facilitate development of Framework standards that are appropriate for the Asia Pacific region. This Framework will serve as a platform upon which country-specific guidelines can be developed or existing guidelines be revised, in a standardised fashion. It is hoped that APCO will serve as an impetus for development of other such alliances world-wide.

\section{NSS44}

ASIA PACIFIC FRAGILITY FRACTURE ALLIANCE: WORKING GROUP OUTPUTS

D.-C. Chan ${ }^{1,2}$

${ }^{1}$ Superintendent, National Taiwan University Hospital ChuTung Branch, Hsinchu, Taiwan, ${ }^{2} \mathrm{Co}-\mathrm{Chair}$, Asia Pacific Fragility Fracture Alliance (APFFA), Hsinchu, Taiwan

The Asia Pacific Fragility Fracture Alliance (APFFA) was launched in November 2018 and is comprised of the following regional and global member organisations:

- Asian Federation of Osteoporosis Societies

- Asia-Oceanian Society of Physical and Rehabilitation Medicine

- Asia Pacific Geriatric Medicine Network

- Asia Pacific Orthopaedic Association

- Fragility Fracture Network

- International Osteoporosis Foundation

- International Society for Clinical Densitometry

The APFFA member organisations signed a multiparty Memorandum of Understanding (MoU) in late 2018. The primary purpose of APFFA is to drive policy change, improve awareness and change political and professional mindsets to facilitate optimal fragility fracture management across the Asia Pacific region. The clinical focus of APFFA is to improve acute care for people in the Asia Pacific region who sustain fragility fractures, subsequent patient rehabilitation and secondary fracture prevention.

Three working groups have been established to develop initiatives concerned with hip fracture registries, education and evidence generation. This presentation will describe current and planned outputs from the working groups.

\section{NSS45}

INFLUENCE OF INITIAL PROTEIN INTAKE ON BONE DENSITY AND ARCHITECTURE IN DYNAPENIC-OBESE OLDER ADULTS

F. Buckinx ${ }^{1}$

${ }^{1}$ University of Liège, Department of Public Health, Epidemiology and Health Economics, Liège, Belgium

Normal aging is associated with muscle mass (sarcopenia) and strength (dynapenia) declines but also with fat mass gain (obesity) which lead to loss of physical performance. Nevertheless, dynapenia combined with obesity increased the risk of falls and loss of mobility than one of these conditions alone. In addition, normal aging leads to bone density loss which is well known to also increased the risk of falls, decline of mobility and fractures. Furthermore, bone architecture seems to be a better predictor of fractures than bone density alone. One potential avenue to counteract these phenomena is to intake a sufficient amount of proteins since protein intake appears to be associated with maintenance of bone density and architecture but also muscle function. More importantly, it seems that the presence of dynapenia, sarcopenia or obesity exacerbate the loss of bone density and architecture and its consequences. However, to our knowledge, the impact of protein intake on OD and bone structure in elderly dynapenic-obese patients is not known. Objective: To compare the influence of protein intake on bone density and architecture in dynapenic-obese older adults.

Methodology: secondary analysis from an observational study. Population: 26 men (M) and women (W) aged (> 60 years), obese (fat mass (\%): $\mathrm{M}>25 ; \mathrm{W}>35$ ) and dynapenic (grip strength $(\mathrm{kg}) /$ body weight $(\mathrm{BW}, \mathrm{kg})$ ): $\mathrm{M}<0.61$; W $<0.44)$ were studied. They were divided a-posteriori into 2 groups according to their initial protein intake: 1) Prot(intake $<1 \mathrm{~g} / \mathrm{kgBW} / \mathrm{d}$ ): $\mathrm{n}=13$ (age: $66.5 \pm 3.3$ years) vs. 2 ) Prot+ (intake $>1.2 \mathrm{~g} / \mathrm{kgBW} / \mathrm{d}$ ): $\mathrm{n}=13$ (age: $67.2 \pm 2.7$ years). Body composition (DXA \& pQCT), femur bone architecture (pQCT), grip strength (lafayette (C), lifestyle habits (Physical activity (7d amrband sensewear@) \& nutrition intake (3-d dairy records using nutrific(@)) were evaluated.

Results: No significant difference was observed on sexe, age, physical activity level but also on muscle, adipose or bone parameters assessed by DXA between our groups. However, the Prot- group has a marrow area $(139 \pm 54$ vs $91 \pm 38 \mathrm{~cm} 2, \mathrm{p}=$ $0.049)$, a total bone area $(650 \pm 69$ vs $579 \pm 91 \mathrm{~cm} 2, \mathrm{p}=0.045)$ but also a compressive loading force (SSI: $3019 \pm 465$ vs 2604 $\pm 560 \mathrm{~cm} 2, \mathrm{p}=0.048)$ significantly greater than the Prot + group.

Conclusion: Surprisingly, lower protein intake appears to protect the bone architecture but not the density in 
dynapenic-obese older adults. These results must be confirmed in a study conducted for this purpose, with a larger sample and an older and / or more fragile population.

\section{NSS46 \\ PRESERVING BODY \& MUSCLE COMPOSITION IN OBESE-OSTEOPENIC OLDER WOMEN: HIGH- INTENSITY INTERVAL TRAINING A POTENTIAL INTERVENTION? \\ M. Aubertin-Leheudre ${ }^{1}$ \\ ${ }^{1}$ Université du Québec à Montréal, Facutlé des Sciences, Dpt des Sc de l'activité Physique \& CRIUGM, Montréal, Canada}

Age-related muscle and bone loss alongside an increase in fat mass can lead to functional decline, an increased risk of falls and fractures, and even, mortality. Highintensity interval training (HIIT) is a promising and time-efficient intervention strategy for maintaining muscle quality and body composition with aging. However, the efficacy of HIIT to improve lean-related muscle mass and adipose tissue distribution in obeseosteopenic older adults is unclear.

Aim: To evaluate the effect of HIIT compared to moderateintensity continuous exercise training (CONT) on body \& muscle composition in obese-osteopenic older women.

Methods: Nineteen obese and osteopenic older women (mean \pm SD age $67.5 \pm 2.7$ years; percent body fat $43.0 \pm 1.3 \%$; number of steps per day $6463 \pm 2619$; areal bone mineral density Tscore <-1 SD) were randomly allocated to a 12-week HIIT group ( $\mathrm{n}=9$; elliptical exercise for 30 seconds at $85 \%$ of agepredicted maximal heart rate [MHR] and 90 seconds at $65 \%$ MHR, 3 times per week for 30 minutes) or a 12-week CONT group ( $\mathrm{n}=10$; treadmill exercise at $65-75 \%$ MHR, 3 times per week for 60 minutes). Body composition (fat-free and fat masses), and muscle composition (Calf muscle crosssectional area $(\mathrm{cm} 2)$ and density $(\mathrm{mg} / \mathrm{cm} 3$; density of tissue within the muscle compartment excluding intramuscular fat and bone areas) and intramuscular and subcutaneous fat areas $(\mathrm{cm} 2)$ ) were measured by DXA and pQCT respectively at baseline and after 12 weeks. Independent t-test analyses compared the mean differences (MD) and $95 \%$ confidence intervals $(95 \% \mathrm{CI})$ for DXA and pQCT outcomes between the HIIT and CONT groups.

Results: On body composition, HIIT improved significantly hip circumference $(112 \pm 15$ to $109 \pm 14 \mathrm{~cm})$ whereas CONT improved leg (12.6 \pm 2.8 to $11.7 \pm 2.6 \mathrm{~kg})$ and appendicular fat $(15.3 \pm 3$ to $14.5 \pm 3.1 \mathrm{~kg})$ masses. Calf muscle area $(\mathrm{MD}=$ $13.99 \mathrm{~cm} 2,95 \%$ CI: $-25.31,-2.67, \mathrm{p}=0.020)$ and subcutaneous fat area (MD $=-22.06 \mathrm{~cm} 2,95 \%$ CI: $-34.96,-9.16$, $\mathrm{p}=0.002$ ) improved in the HIIT group relative to the CONT group. However, there were no between-group differences for calf muscle density $(\mathrm{MD}=-3.00 \mathrm{mg} / \mathrm{cm} 3,95 \% \mathrm{CI}:-6.27$, $0.27, \mathrm{p}=0.069)$ and intramuscular fat area $(\mathrm{MD}=-1.31,95 \%$
CI $-3.81,1.19, \mathrm{p}=0.283$ ). Percent adherence was $86 \%$ and 97\% in the HIIT and CONT groups, respectively.

Conclusions: HIIT may represent a promising intervention strategy for improving lean muscle and adipose tissue properties in obese-osteopenic older adults compared to traditional continuous exercise training approaches. Further investigation is needed to confirm the clinical relevance of our pilot trial results for falls and fracture prevention.

\section{NSS47}

PHYSICAL PERFORMANCE IN OBESEOSTEOPENIC ELDERLY WOMEN: HIGHINTENSITY INTERVAL TRAINING MORE EFFICIENT THAN CONTINUOUS AEROBIC TRAINING?

B. Fougère $^{1}$

${ }^{1}$ Division of Geriatric Medicine, Tours University Hospital, Tours, France

Ageing is associated with functional incapacities which lead to falls, loss of autonomy and mortality. Being obese and osteopenic seems to worsen physical health more than each of these conditions alone. Physical activity (aerobic training) is recognized to be an efficient strategy to improve many health-related outcomes.

Objective: We aimed to compare the effect of high-intensity interval training (HIIT) and moderate-intensity continuous training (CONT) on physical performance in obeseosteopenic elderly women.

Methods: Nineteen inactive $(<10,000$ steps/d) elderly $(67$ $\pm 3 y$ ), obese (fat mass: $43 \pm 6 \%$ ) and osteopenic (BMD $<-1 \mathrm{~T}$ score) women were randomly divided into 2 groups and completed a 12 week exercise intervention: HIIT ( $\mathrm{n}=9$, elliptical device; cycle: $30 \mathrm{sec}$ at $85 \%$ and $90 \mathrm{sec}$ at $65 \%$ of maximal age-predicted heart rate; $3 \times 30 \mathrm{~min} /$ week) and CONT $(\mathrm{n}=10$, treadmill at $65-75 \%$ maximal age-predicted heart rate; $3 \times 1 \mathrm{~h} /$ week). Upper (hand dynamometer) and lower (KES) muscle strength, muscle power (leg power rig) and functional capacities (4m and 6min-6MWT-walking test, chair and step tests) were measured pre and post-intervention. $p<0.05$ was considered significant.

Results: On physical performance, HIIT improved significantly leg power ( $102 \pm 26$ to $137 \pm 30$ Watt), step test ( $28 \pm 3$ to $34 \pm 3$ ) and chair test ( $19 \pm 3$ to $16 \pm 4 \mathrm{~s}$ ) whereas CONT improved handgrip strength ( $21.6 \pm 4.4$ to $24.2 \pm 5.4 \mathrm{~kg})$. Only $6 \mathrm{MWT}$ was increased in both groups $(+16 \%$ in HIIT and $+6 \%$ in CONT).

Conclusion: HIIT, at half the training volume, seems more effective to improve physical performance in inactive obeseosteopenic elderly women than CONT aerobic exercise. Clinicians should consider HIIT as a strategy to prevent physical disability in obese-osteopenic women, although further investigations with larger sample sizes are still needed to confirm our findings. 


\section{NSS48}

\section{ROLE OF ZINC IN OSTEOPOROSIS AND VASCULAR CALCIFICATION: KILLING 2 BIRDS WITH 1 STONE?} W. Chen ${ }^{1}$

${ }^{1}$ Assistant Professor of Medicine, Albert Einstein College of Medicine, New-York, United States

Bone mineralization and vascular calcification share many common pathways in pathophysiology. This creates a calcification paradox: patients with low bone mineral density are more likely to have vascular calcification and calcium supplementation used to treat osteoporosis may worsen vascular calcification. Zinc, an essential mineral, has been shown to prevent osteoporosis by stimulating osteoblastic and inhibiting osteoclastic activity. Recently, zinc has also been shown to protect against vascular calcification. Thus, zinc supplementation is a potential therapeutic strategy for both osteoporosis and vascular calcification. Furthermore, research on zinc, bone mineralization and vascular calcification can provide insights into the calcification paradox.

Learning objective:

1) To understand calcification paradox in bone and vascular disease and associated therapeutic dilemma

2) To discuss the relationship of zinc with osteoporosis and vascular calcification

3) To examine the mechanisms of zinc in preventing osteoporosis and protecting against vascular calcification

4) To discuss the potential therapeutic value of zinc for both osteoporosis and vascular calcification

\section{NSS49 \\ BONE, SKELETAL MUSCLE, AND SARCOPENIA IN CHRONIC KIDNEY DISEASE}

M. Abramowitz ${ }^{1}$

${ }^{1}$ Associate Professor of Medicine, Albert Einstein College of Medicine, New-York, United States

Patients with chronic kidney disease (CKD) experience not only bone disease but skeletal muscle dysfunction as well. Sarcopenia, defined as low muscle mass and poor physical function, is common in CKD, and is associated with poor outcomes, including increased risk of fractures. A number of metabolic and hormonal alterations in CKD affect skeletal muscle health; many are factors that also cause bone pathology. Furthermore, there is substantial overlap between the disturbances seen in CKD and those in aging. This presentation will focus on the mechanisms and clinical impact of sarcopenia in adults with CKD.

Learning objectives:

1) To understand the common factors affecting both bone and skeletal muscle in patients with CKD.
2) To recognize CKD as a state of accelerated aging.

3) To understand the impact of sarcopenia on clinical and patient-oriented outcomes in CKD.

4) To discuss well-recognized and newly identified mechanisms of skeletal muscle dysfunction and physical function impairment in CKD.

\section{NSS50}

\section{TREATING BONE DISEASE IN THE WOMAN WITH CHRONIC KIDNEY DISEASE}

M. Coco ${ }^{1}$

${ }^{1}$ Professor of Medicine, Albert Einstein College of Medicine, Montefiore Medical Center, New-York, United States

Women are especially vulnerable to bone disease. There is a natural decline in bone preserving hormones with age leading to osteoporosis. Women with Chronic Kidney Disease are further subjected to added stress on their bone health. Hyperparathyroidism with ensuing calcium and phosphorus derangements will decrease the quality of bone. If the woman undergoes renal transplantation, another layer of bone stressors is added attributable to immunosuppressive agents. The end result is a decrease in bone mineral density and increased risk of bone fractures. Standard of care treatment in the general population with osteoporosis may not necessarily benefit these women.

Learning objectives.

1. To review the types of metabolic bone disease that can develop in the woman with CKD, and suggestions to tailor treatment that enhance bone quality.

2. To review the role of DEXA, FRAX modeling, biochemical bone turnover parameters and bone biopsy in diagnosing underlying bone disease.

3. To review the effects of immunosuppression used to treat underlying renal disease and renal transplantation on bone health of women.

4. To discuss the treatment of osteoporosis in the woman with CKD: the roles of antiresorptive and anabolic therapies.

\section{NSS51}

QUALITY OF LIFE IN SARCOPENIA: AN UPDATE C. Beaudart ${ }^{1,2}$

${ }^{1}$ Department of Public Health, Epidemiology and Health Economics, University of Liège, Liège, Belgium, ${ }^{2} \mathrm{WHO}$ Collaborating Center for Public Health Aspects of Musculoskeletal Health and Ageing, Liège, Belgium

The association between sarcopenia and quality of life became a topic of high interest in the past 10 last years. The prospective and generalized loss of muscle mass, muscle strength, physical performance and muscle quality resulting from 
sarcopenia could emerge as mobility impairments, malnutrition, disability and increased sedentary behaviour. These symptoms could themselves lead to a loss of independence, a higher risk of falls, fractures, hospital or nursing home admissions and/or an impaired ability to perform daily activities. All of these factors contribute to a lower QoL in sarcopenia. Evidence from the literature, issued from a literature review performed in July 2019, underlines that age-related sarcopenia seems to be related to poorer quality of life. Across the 35 original studies identified throughout our review, a large majority of studies showed a lower quality of life, in specific domains or as a whole, for sarcopenic individuals compared to non-sarcopenic ones. Quality of life seems mainly to be impacted on physical and functional domains, suggesting that specific QoL questionnaires for sarcopenia could be of interest.

\section{NSS52}

\section{OVERVIEW OF GENERIC AND SPECIFIC PATIENT REPORTED OUTCOME MEASURES (PROMS) IN SARCOPENIA}

A. Geerinck $^{1,2}$

${ }^{1}$ Department of Public Health, Epidemiology and Health Economics, University of Liège, Liège, Belgium, ${ }^{2} \mathrm{WHO}$ Collaborating Center for Public Health Aspects of Musculoskeletal Health and Ageing, Liège, Belgium

To measure patients' experiences and perspectives in sarcopenia, valid and reliable outcome measures are needed. Unfortunately, few PROMs have been validated for use in sarcopenic subjects, and even fewer have been specifically designed to capture the impact of sarcopenia on the patients' own experience. There are two instruments that fit these criteria: the Age-Related Muscle Loss Questionnaire (ARMLQ, also referred to as SarcoPRO®) which measures the functional impacts of reduced muscle strength, and the Sarcopenia Quality of Life (SarQoL®) questionnaire which evaluates healthrelated quality of life. A project is also currently underway to obtain FDA recognition for the PROMIS ${ }^{\circledR}$ Physical Functioning item bank as a qualified clinical outcome assessment in sarcopenia. Generic measures, i.e. those not specifically designed for use in sarcopenia, can also provide valuable information, because the data they provide can be compared across populations. Popular measures in this category are the Short-Form 36-item (SF-36) questionnaire and the EuroQoL 5dimension (EQ-5D) and the associated visual analogue scale. However, no investigations of the psychometric properties of both questionnaires have been performed for use in sarcopenia. In conclusion, few tools are available that have demonstrated to be appropriate, valid and reliable for sarcopenic samples. Because of this, generic measures are often used.

\section{NSS53}

VERTEBRAL FRACTURE FOLLOWING D-MAB DISCONTINUATION: SUPPORTING DATA

\section{O. Rosero Olarte ${ }^{1,2,3,4}$}

${ }^{1}$ Asociación Colombiana de Endocrinologia - ACE, Bogotá, Colombia, ${ }^{2} \mathrm{ACE}$, Asociación Colombiana de Osteoporosis y Metabolismo Mineral - ACOMM, Bogotá, Colombia, ${ }^{3}$ Endocrine Society, Washington, United States, ${ }^{4}$ American Association of Clinical Endocrinology Member, Jacksonville, United States

Denosumab (DMAB), is a fully human monoclonal antibody that exerts its potent antiresorptive action via neutralization of RANK ligand to effectively inhibit bone remodeling. It has been marketed for treatment of osteoporosis since 2010. DMAB reduces bone resorption and improves bone mineral density (BMD) ${ }^{1}$. DMAB inhibits bone resorption in a potent manner, as evident from a profound drop (up to $80 \%$ ) in bone turnover markers (BTM). Unlike bisphosphonates (BP), DMAB is not incorporated into bone. Therefore, it's effect on bone resorption ceases rapidly after treatment discontinuation $^{2}$. BTM suppression vanishes away after 6 months past one single injection, demonstrating DMAB reversibility. Furthermore, upon medication discontinuation, a remarkable rebound rise of BTM occurs $^{3}$. It has been widely demonstrated that the rebound effect magnitude might be influenced by prior bisphosphonate treatment, with a lesser degree of C-telopeptide elevation upon DMAB discontinuation in patients previously treated with BP. There is limited information from clinical trials about fracture risk upon stopping DMAB therapy. Brown and colleagues reported no increase in vertebral fracture incidence in 1001 patients who discontinued DMAB or placebo in the FREEDOM and FREEDOM Extension trials. However, among subjects off-treatment there was an excess of multiple vertebral fractures in DMAB Discontinuers (DD) compared to those that discontinued placebo ${ }^{4}$. In the real world data publication, a total of 1,500 DD (92\% females, age 71.8 \pm 9.5 y.o.) was compared to 1,610 persistent users $(91 \%$ females, age $71.7 \pm 8.8$ y.o.). At baseline, the groups were comparable in fracture history, BP exposure, smoking, and bone mineral density (BMD). Multiple vertebral fracture (MVF) occurred in $12(0.8 \%)$ DD compared to $02(0.1 \%)$ patients who were persistent users $(p=0.006)$. The overall rate of fractures per 100 patient-years of follow-up was significantly higher in DD than persistent users (RR 3.2, 95\% CI 2.2-4.8), as well as the rate of vertebral fractures (RR 4.7, 95\% CI 2.3-9.6) and MVF (RR 14.6, 95\% CI 3.3-65.3) Conclusion: relevant data supports the high risk of MVF after stopping treatment with DMAB. 


\section{References}

1. Cummings SR, San Martin J, McClung MR, Siris ES, Eastell R, Reid IR, Delmas P, Zoog HB, Austin M, Wang A, Kutilek S, Adami S, Zanchetta J, Libanati C, Siddhanti S, Christiansen C; FREEDOM Trial. Denosumab for prevention of fractures in postmenopausal women with osteoporosis. N Engl J Med. 2009; 361(8):756-65.

2. Lewiecki EM1, Miller PD, McClung MR, Cohen SB, Bolognese MA, Liu Y, Wang A, Siddhanti S, Fitzpatrick LA; AMG 162 Bone Loss Study Group. Two-year treatment with denosumab (AMG 162) in a randomized phase 2 study of postmenopausal women with low BMD. J Bone Miner Res. 2007; 22(12):183241.

3. Bone HG, Bolognese MA, Yuen CK, Kendler DL, Miller PD, Yang YC, Grazette L, San Martin J, Gallagher JC. Effects of denosumab treatment and discontinuation on bone mineral density and bone turnover markers in postmenopausal women with low bone mass. J Clin Endocrinol Metab. 2011; 96(4):972-80.

4. Brown JP, Roux C, Törring O, Ho PR, Beck Jensen JE, Gilchrist N, Recknor C, Austin M, Wang A, Grauer A, Wagman RB. Discontinuation of denosumab and associated fracture incidence: analysis from the Fracture Reduction Evaluation of Denosumab in Osteoporosis Every 6 Months (FREEDOM) trial. J Bone Miner Res. 2013; 28(4): 746-52.

5. Tripto-Shkolnik L, Fund N, Rouach V, Chodick G, Shalev V, Goldshtein I. Fracture incidence after denosumab discontinuation: Real-world data from a large healthcare provider. Bone 2020; 130: 115150.

\section{NSS54 \\ PATHOPHYSIOLOGY OF THE OSTEOCLASTIC "FLARE" AFTER DISCONTINUATION}

\section{A. Medina Orjuella ${ }^{1,2,3}$}

${ }^{1}$ Internal Medicine \& Endocrinology Specialist at Fundación Universitaria de Ciencias de la Salud. Universidad Nacional de Colombia, Bogotá, Colombia, ${ }^{2}$ Asociación Colombiana de Endocrinologia - ACE, Bogotá, Colombia, ${ }^{3}$ Asociación Colombiana de Osteoporosis y Metabolismo Mineral ACOMM, Bogotá, Colombia

Denosumab (DMAB) is a human monoclonal antibody that specifically binds to receptor activator of NF-kB ligand (RANKL) reversibly blocking bone resorption, leading to decreased bone turnover markers (BTMs), increasing bone mineral density (BMD), and reducing fracture risk. The activation of the receptor activator of NF-kB (RANK) by its ligand (RANKL), leads to maturation from pre-osteoclasts, activity, and survival of osteoclasts ${ }^{1}$. After two years of continuous treatment with DMAB every 6 months, or $30 \mathrm{mg}$ every 3 months, for postmenopausal women, it was observed an increased BMD at the lumbar spine $(9.4 \%$ to $11.8 \%)$ and total hip $(4.0 \%$ to $6.1 \%)$. BTMs were consistently suppressed over 48 months. Discontinuation of DMAB was associated with a BMD decrease of $6.6 \%$ at the lumbar spine and $5.3 \%$ at the total hip within the first 12 months. Serum C-telopeptide (CTX) levels increased to twice the placebo and returned to values near baseline ${ }^{2}$. Possible explanations for the rebound effect could be that an increased pool of osteoclast precursors which were dormant during the treatment period with DMAB, become activated after its discontinuation, also there is a high RANKL/osteoprotegerin ratio after DMAB, that is cleared from circulation, leading to a rapid rebound in remodeling rates $^{3}$. Following the biomechanical of skeleton, individuals that have a preset level of bone density and remodeling that have been influenced by external conditions, in this case, a medication (DMAB), tend to return to baseline level, increasing bone turnover and decreasing $\mathrm{BMD}^{2}$. In the case of BTMs, the rebound effect is more prominent as the duration of DMAB treatment increases, and the reversal of changes in BMD may be related to the duration of DMAB therapy and the magnitude of bone loss may be related to the amount of BMD gained during treatment; although it is necessary to prove this by extension trials ${ }^{1-4}$. The multiple vertebral fractures in the reported cases are the result of the high level of bone remodeling after withdrawal of reversible antiresorptives like DMAB, which leads to bone resorption in a tissue that was continuously exposed to low accumulation of fatigue damage but without repair during therapy, then, high rate of bone turnover and bone loss developed during the rebound would lead to a high rate of damage accumulation and a shortened vertebral fatigue life, nevertheless, there is little information about the effects of withdrawal after longer-term DMAB treatment when BMD gains on therapy ${ }^{5}$. The association between vertebral fractures and high resorption and decreased $\mathrm{BMD}$, is observed in early menopause, where there is a rapid bone loss that will impact in older postmenopausal women who are losing bone less rapidly, with more rapid destruction of trabecular microarchitecture, so the fracture risk is directly related to bone turnover in untreated postmenopausal women who are losing bone mass at a modest rate ${ }^{5}$. Other antiresorptive therapies that do not persist in bone, including estrogen therapy and estrogen receptor agonists/antagonists, have the same reversible effect on BMD and BMT when discounted as DMAB, returning to pretreatment levels of BMD and BTM. Despite returning to pretreatment levels, large observational studies of postmenopausal estrogen therapy discontinuation have not shown an increase in fracture risk as it occurs with DMAB. Reversibility of BMD and BTM levels have also been observed with teriparatide ${ }^{6}$. In the Frost mechanostat theory based on Wolff's Law, the bone tends to return to the basal condition after removing the agent that changed it ${ }^{7}$. 


\section{References}

1. Tsourdi E, Langdahl B, Cohen-Solal M, Aubry-Rozier B, Fink-Eriksen E, Guañabens N, Obermayer-Pietsch B, Ralstonj SH, Eastell R, Carola M. Discontinuation of Denosumab therapy for osteoporosis: a systematic review and position statement by ECTS. Bone 105 (2017) 11-17.

2. Miller PD, Bolognese MA, Lewiecki EM, McClung MR, Ding B, Austin M, Liu Y, San Martin J. Effect of denosumab on bone density and turnover in postmenopausal women with low bone mass after long-term continued, discontinued, and restarting of therapy: a randomized blinded phase 2 clinical trial for the AMG 162 Bone Loss Study Group Bone 2008; 43: 222-9.

3. Bone HG, Bolognese MA, Yuen CK, Kendler DL, Wang H, Liu Y, San Martin J. Effects of denosumab on bone mineral density and bone turnover postmenopausal women. J Clin Endocrinol Metab. 2008; 93:2149-57.

4. McClung MR. Cancel the denosumab holiday. Osteoporos Int. 2016; 27: 1677-82.

5. Popp AW, Zysset PK, Lippuner K. Rebound-associated vertebral fractures after discontinuation of denosumabfrom clinic and biomechanics. Osteoporos Int. 2016; 27(5):1917-21.

6. Bone HG, Bolognese MA, Yuen CK, Kendler DL, Miller PD, Yang YC, Grazette L, San Martin J, Gallagher JC. Effects of denosumab treatment and discontinuation on bone mineral density and bone turnover markers in postmenopausal women with low bone mass. J Clin Endocrinol Metab. 2011 Apr;96(4):972-80.

7. Frost, H. From Wolff's Law to the mechanostato: a new "face" of physiology. J Orthop Sci. 1998; 3:282-6.

\section{NSS55 \\ BIOCHEMICHAL AND IMAGING ASSESSMENT: UPS AND DOWNS \\ $\underline{\text { B. Muzzi Camargos }}^{1,2,3}$ \\ ${ }^{1}$ Bone Densitometry and Metabolism Unit Coordinator at Densimater - Rede Mater Dei de Saúde, Belo Horizonte, Brazil, ${ }^{2}$ Vice President for Densitometry at Sociedade de Radiologia de Minas Gerais, Minas Gerais, Brazil, ${ }^{3} \mathrm{IOF}$ Committee of Scientific Advisor Member, Belo Horizonte, Brazil}

Treat-to-target approach has helped clinicians to plan osteoporosis treatment for their patients ${ }^{1}$.

This approach is also applied to other chronic diseases like hypertension $^{2}$ diabetes mellitus ${ }^{3}$ and rheumatoid arthritis ${ }^{4}$.

Osteoporosis treatment usually begins with an oral bisphosphonate (alendronate) if there is no contraindication. The patient is monitored and the response to treatment is often defined as stability or an increase in bone mineral density (BMD) and decrease in bone turnover markers (BTMs) in the absence of fractures ${ }^{5}$. Treatment failure was addressed by The International Osteoporosis Foundation and three different situations were defined: 1) two or more incident fractures during treatment; 2) one incident fracture with elevated bone turnover marker at baseline without significant decrease with antiresorptive therapy and/or a significant decrease in BMD; 3) no significant decrease in bone turnover marker and a significant decrease in $\mathrm{BMD}^{6}$. If the patient is responding adequately to bisphosphonates, the treatment can be maintained for 3-5 years if the fracture risk is low. Then, a drug holiday can be proposed ${ }^{7}$. If fracture risk is high after therapy for 3-5 years, treatment may be continued up to 10 years. There is no evidence strong enough to support treatment beyond that period of time. The drug holiday is possible due to long-lasting bisphosphonate effect within the skeleton. But the holiday approach is not appropriate with nonbisphosphonate drugs, because they lose their effectiveness very rapidly after discontinuation ${ }^{8}$. This is critically important for DMAB therapy. In the absence of fractures to define who must continue under treatment or not, surrogate markers of fracture risk shall be used. The most reliable ones are: bone turnover markers (BTMs), fracture risk assessment algorithms and $\mathrm{BMD}^{9}$. Continuing alendronate therapy in women with low risk (femoral neck $\mathrm{T}$-scores $\leq-2.5$ and no prevalent vertebral fractures) reduced the incidence of nonvertebral fractures. But no further reduction of nonvertebral fracture risk was observed in women with femoral neck T-scores above $-2.0^{10}$. Similar results were found with another bisphosphonate: zoledronic acid. Post-hoc analysis of the HORIZON-PFT found that women having a femur $\mathrm{T}$ - score $\leq-2.5$ were at greater risk of morphometric vertebral fractures and nonvertebral fractures when treatment was discontinued, compared with continuing treatment up to six years ${ }^{11}$. For patients under osteoporosis treatment, larger $\mathrm{BMD}$ increases reflected in greater reductions of risk for nonvertebral fractures. Different analyses demonstrated that it is true not only for denosumab (DMAB) patients in the FREEDOM extension study ${ }^{12}$, but also for other agents as well $^{13}$. More recently, an Official Position paper from International Society for Clinical Densitometry recommended that serial BMD testing should be used to monitor individuals following cessation of osteoporosis pharmacologic therapy ${ }^{14}$. All the information above can lead us to - at least - two major conclusions regarding DMAB and BMD monitoring: 1) BMD measured by DXA is still the most accurate imaging diagnostic surrogate marker for initiating or stopping therapy in the absence of fractures, 2) as treatment stops, BMD falls much more rapidly for patients on DMAB than for those under bisphosphonate therapy.

Bone Turnover Markers (BTMs) 
Discontinuation of DMAB therapy is followed by a transient increase of bone turnover markers (BTMs) above pretreatment values, together with accelerated bone loss, and potentially an increased risk of multiple vertebral fractures ${ }^{15}$.

Even if drug holiday is not recommended for DMAB patients, those experiencing BMD gains and at low risk of developing osteoporotic fractures after 5 years of therapy; a decision to discontinue DMAB could be made. If that is the case, bisphosphonate therapy should be considered to reduce or prevent the rebound increase in bone turnover ${ }^{16}$.

BTMs (CTX) at 9 and 12 months after the last injection of DMAB showed a significant increase values above pretreatment values and a progressive decrease thereafter; showing a significant correlation between the time since last DMAB injection and serum CTX values in all patients who were treated with denosumab independently of the presence of vertebral fractures. This assumed initial increase in RANKL is responsible for the dramatic increase in osteoclastogenesis evidenced by the 13fold increase in levels of RANKL mRNA followed by an increased activity of osteoclasts demonstrated by the upregulation of cathepsin $\mathrm{K}$ mRNA ${ }^{17}$.

Although the optimal bisphosphonate regimen post-DMAB is currently unknown, cessation of DMAB do happen on real world population and should be tightly monitored once the risk of multiple fractures after discontinuation has been documented on quite a few settings. BMD measurements performed by DXA are not widely covered by healthcare providers for assessment intervals shorter than one year. Therefore, BTMs arise as early surrogate markers of a potential osteoclastic rebound before BMD changes can be assessed.

\section{References}

1. Lewiecki EM. Osteoporosis: Treat-to-Target. Curr Osteoporos Rep. 2017; 15:103-9.

2. James PA, Oparil S, Carter BL, Cushman WC, Dennison- Himmelfarb C, Handler J, et al. 2014 evidence-based guideline for the management of high blood pressure in adults: report from the panel members appointed to the eighth joint National Committee (JNC 8). JAMA. 2014;311(5):507-20.

3. Chamberlain JJ, Rhinehart AS, Shaefer Jr CF, Neuman A. Diagnosis and Management of Diabetes: synopsis of the 2016 American Diabetes Association standards of medical Care in Diabetes. Ann Intern Med. 2016;164(8):542-52.

4. Singh JA, Saag KG, Bridges Jr SL, Akl EA, Bannuru RR, Sullivan MC, et al. 2015 American College of Rheumatology Guideline for the treatment of rheumatoid arthritis. Arthritis Rheumatol. 2016;68(1):1-26.

5. Lewiecki EM, Watts NB. Assessing response to osteoporosis therapy. Osteoporos Int. 2008;19(10):1363-8.
6. Diez-Perez A, Adachi JD, Agnusdei D, Bilezikian JP, Compston JE, Cummings SR, et al. Treatment failure in osteoporosis. Osteoporos Int. 2012;23:2769-74.

7. McClung M, Harris ST, Miller PD, Bauer DC, Davison KS, Dian L, et al. Bisphosphonate therapy for osteoporosis: benefits, risks, and drug holiday. Am J Med. 2013;126(1):13-20.

8. McClung MR. Cancel the denosumab holiday. Osteoporos Int. 2016;27(5):1677-82.

9. Cummings SR, Cosman F, Eastell R, Reid IR, Mehta M, Lewiecki EM. Goal-directed treatment of osteoporosis. J Bone Miner Res. 2013;28(3):433-8.

10. Schwartz AV, Bauer DC, Cummings SR, Cauley JA, Ensrud KE, Palermo L, et al. Efficacy of continued alendronate for fractures in women with and without prevalent vertebral fracture: the FLEX trial. J Bone Miner Res. 2010;25(5):976-82.

11. Cosman F, Cauley JA, Eastell R, Boonen S, Palermo L, Reid IR, et al. Reassessment of fracture risk in women after 3 years of treat- ment with zoledronic acid: when is it reasonable to discontinue treatment? J Clin Endocrinol Metab. 2014;99(12):4546-54.

12. Ferrari S, Libanati C, Lin CJF, Adami S, Brown JP, Cosman F, et al. Relationship between total hip BMD $\mathrm{T}$-score and incidence of nonvertebral fracture with up to 8 years of denosumab treatment. J Bone Miner Res. 2015;30(Suppl. 1):S49. This is a report that larger increases in BMD with denosumab are associated with a greater reduction in fracture risk.

13. Black DM, Vittinghoff E, Eastell R, Bouxsein M, McCulloch C, Cawthon PM, et al. Hip BMD by DXA can reliably estimate reduction in hip risk in osteoporosis trials: a meta-regression. J Bone Miner Res. 2015;30(S1):S49.

14. Repeating Measurement of Bone Mineral Density when Monitoring with Dual-energy X-ray Absorptiometry: 2019 ISCD Official Position. Kendler DL, Compston J, Carey JJ, Wu CH, Ibrahim A, Lewiecki EM. J Clin Densitom. 2019; 22(4):489-500.

15. Uebelhart B, Rizzoli R, Ferrari SL. Retrospective evaluation of serum CTX levels after denosumab discontinuation in patients with or without prior exposure to bisphosphonates. Osteoporos Int. 2017;9:2701-05.

16. Tsourdi E, Langdahl B, Cohen-Solal M, Aubry-Rozier B, Eriksen EF, Guañabens N, Obermayer-Pietsch B, Ralston SH, Eastell R, Zillikens MC. Discontinuation of Denosumab therapy for osteoporosis: A systematic review and position statement by ECTS. Bone. 2017;105:11-17.

17. Anastasilakis AD, Yavropoulou MP, Makras P, Sakellariou GT, Papadopoulou F, Gerou S, Papapoulos SE. Increased osteoclastogenesis in patients with vertebral fractures following discontinuation of 
denosumab treatment. European Journal of Endocrinology 2017; 176, 677-83.

\section{NSS56 \\ BONE LOSS AFTER D-MAB DISCONTINUATION IN 150 PATIENTS}

M. Belen Zanchetta ${ }^{1,2}$

${ }^{1}$ Directora de la Maestría de Osteología y Metabolismo Mineral, Universidad del Salvador, Buenos Aires, Argentina, ${ }^{2}$ Medical Director at Instituto de Diagnóstico e Investigaciones Metabólicas - IDIM, Buenos Aires, Argentina

DMAB discontinuation is associated with the reversal of the effects attained with treatment. Bisphosphonates administration has been recommended to avoid rapid bone loss but few studies have addressed this question. Preliminary clinical experience will be presented on BMD and bone turnover markers between postmenopausal women $(n=98)$ who received bisphosphonates after stopping DMAB and those who not. There were no significant differences in age, height, weight or BMI between the groups. Mean treatment time with DMAB was 2.33 years. In the non-treated group we observed a significant reduction in $\operatorname{LS} \operatorname{BMD}(-4.1 \pm 6 \% ; \mathrm{p}<0.01)$ and FN BMD $(-3.3 \pm 5.4 \%(\mathrm{p}<0.01)$. In the treated-group, LS BMD was significantly lower than baseline $(3.3 \pm 4.5 \%$, $\mathrm{p}=0.05)$ but FN BMD was non-significantly lower $(0.1 \pm 4.3$ $\%, \mathrm{p}=0.25$ ). After stopping DMAB, bone turnover markers (CTX and osteocalcin) increased significantly in both groups but to a much lesser extend in the treated-group, these differences between groups were statistically significant. No patients in the treated-group had fragility fractures during follow-up but two patients in the non-treated group had vertebral fractures. We observed that bisphosphonates ameliorated bone loss, especially in the hip, and prevented the high rebound of bone remodeling markers after DMAB discontinuation. It is essential to enhance physicians' awareness of the need to start bisphophonates after discontinuing DMAB.

\section{NSS57}

\section{D-MAB PATIENT MANAGEMENT IN THE} CLINICAL SETTING: STEPPING ON THE RIGHT FOOT

\section{F. Vidal Neira ${ }^{1,2,3}$}

${ }^{1}$ Specialist in Rheumatology. Head of the Rheumatology Department at Hospital María Auxiliadora, Lima, Peru,

${ }^{2}$ Medical Director at Centro Diagnóstico de la Osteoporosis y Enfermedades Reumáticas - CEDOR, Lima, Peru, ${ }^{3} \mathrm{IOF}$ Regional Advisory Councils, Region Andina, Colombia

The first aspect to consider is when to start treatment with DMAB, specially in treatment-naïve osteoporotic women. Most guidelines recommend oral bisphosphonates as first- line medications and consider DMAB as an alternative initial therapy, in particular for patients at high risk of non-vertebral and hip fractures ${ }^{1-3}$. Moreover, in certain countries DMAB is reimbursed only when used after a bisphosphonate ${ }^{1}$. However, the patient profile to whom DMAB would be a better first line choice it is still unclear. Some clinical situations in which DMAB can be considered instead of bisphosphonates are patients with severe renal impairment or when these medications have been ineffective or are not well tolerated $^{4,5}$. Before starting DMAB, certain considerations must be taken. DMAB can lead to a reduction in serum calcium concentration. For this reason, it is important to ensure that vitamin D levels and calcium intake are adequate ${ }^{5}$. Osteonecrosis of the jaw is a serious adverse event of some antiresorptive medications like DMAB. A dental evaluation should be performed routinely before initiating therapy and implement a dental care plan while on the drug is also recommended $^{6,7}$. Continuous administration of DMAB is associated with gain of bone mineral density (BMD) over 10 years and reduced risk of fractures ${ }^{8}$. Discontinuing the drug specially after the second dose is associated with a "rebound effect" characterized by increase in bone turnover, loss of BMD and increased risk of vertebral fractures, so that certain considerations must be taken before the DMAB discontinuation ${ }^{9}, 10$. Prevalent vertebral fractures are considered the most important risk factor for the development of new clinical vertebral fractures following DMAB cessation and it is wise to at perform a spine $\mathrm{x}$-ray at the time of discontinuing DMAB ${ }^{11,12}$. It has been proposed that the use of a potent bisphosphonate (zoledronic acid or alendronate) after discontinuing DMAB could prevent or mitigate the rebound effect ${ }^{9-12}$. However, the timeframe for initiation and duration of treatment are not yet clarified. This review will further discuss the available current evidence regarding the specifics of using bisphosphonates in the setting of discontinuing DMAB.

\section{References}

1. Kanis JA, Cooper C, Rizzoli R, Reginster JY. European guidance for the diagnosis and management of osteoporosis in postmenopausal women. Osteoporosis Int. 2019; 30(1):3-44.

2. Eastell R, Rosen CJ, Black DM, Cheung AM, Murad M, Shoback D. Pharmacological management of osteoporosis in postmenopausal women: an Endocrine Society Clinical Practice Guideline. J Clin Endocr Metab 2019;104(5):1595-1622.

3. Briot K, Roux C, Thomas T, Blain H, Buchon D, Chapurlat R, et al. 2018 update of French recommendations on the management of postmenopausal osteoporosis. Joint Bone Spine. 2018;85(5):519-530.

4. Anastasilakis AD, Polyzos SA, Makras P. Denosumab vs bisphosphonates for the treatment of postmenopausal osteoporosis. Eur J Endocrinol. 2018;179: R31-R45 
5. Wilcock A, Charlesworth S, Toller CS, Girish R, Mihalyo M, Howard P. Denosumab: AHFS 92: 24. J Pain Symptom Manag. 2018:56(2): 295-301.

6. Bugueno JM, Migliorati CA. The American Academy of Oral Medicine clinical practice statement: dental care for the patient on antiresorptive drug therapy. Oral Surg, Oral Med O. 2019;127(2), 136-139.

7. Watts NB, Grbic JT, Binkley N, Papapoulos S, Butler $\mathrm{PW}$, Y in $\mathrm{X}$, et al. Invasive oral procedures and events in postmenopausal women with osteoporosis treated with denosumab for up to 10 years. J Clin Endocr Metab 2019;104(6): 2443-2452.

8. Bone HG, Wagman RB, Brandi ML, Brown JP, Chapurlat R, Cummings SR, et al. 10 years of denosumab treatment in postmenopausal women with osteoporosis: results from the phase 3 randomised FREEDOM trial and open-label extension. The Lancet Diabetes Endo. 2017; 5(7):513-523.

9. Lamy O, Stoll D, Aubry-Rozier B, Rodriguez EG. Stopping denosumab. Curr Osteoporos Rep. 2019;17(1): 8-15.

10. Tsourdi E, Langdahl B, Cohen-Solal M, Aubry-Rozier B, Eriksen EF, Guañabens N, et al. Discontinuation of denosumab therapy for osteoporosis: a systematic review and position statement by ECTS. Bone. 2017; 105:11-17.

11. Cummings SR, Ferrari S, Eastell R, Gilchrist N, Jensen JB, McClung M, et al. Vertebral fractures after discontinuation of denosumab: a post hoc analysis of the randomized placebo-controlled FREEDOM trial and its extension. J Bone Miner Res. 2018;33(2):190-198.

12. Anastasilakis AD, Papapoulos SE, Polyzos SA, Appelman-Dijkstra NM, Makras P. Zoledronate for the Prevention of Bone Loss in Women Discontinuing Denosumab Treatment. A Prospective 2-year Clinical Trial. J Bone Miner Res. 2019;34(12): 2220-2228.

\section{NSS58}

INTRODUCTION "WHEN A DXA IS INDICATED, A VFA IS ALSO RECOMMENDED”

W. F. Lems ${ }^{1}$

${ }^{1}$ VU Medical Centre, Amsterdam Rheumatology and Immunology Center, Amsterdam, The Netherlands

There are world-wide large diagnostic and treatment gaps in the field of osteoporosis, leading to a low percentage of patients adequately diagnosed and treated for osteoporosis versus the total number of osteoporotic patients. One of the most powerfull instruments to diagnose osteoporosis is the Fracture Liaison Service (FLS), in which elderly patients with a recent fracture are screened for underlying low BMD of the lumbar spine and/or hips with a DXA. In this minisymposium we will discuss the clinical consequences of additionally testing for vertebral fractures by systematically performing Vertebral Fracture Assesment (VFA) in all patients visiting the FLS. In elderly patients with a recent fracture and a BMD in the osteoporotic range, the finding of a vertebral fracture does usually not influence the start of anti-osteoporotic drug-treatment, while diagnosing one or more vertebral fractures in an elderly patient with osteopenia can be an indication for initiating anti-osteoporotic drug-treatment. Thus, it is plausible that this will lead to less underdiagnosis in patients at high risk for future fractures, and as a consequence, more adequate treatment in patients visiting the FLS.

The IOF Capture the Fracture $(\mathrm{CtF})$ initiative helps clinicians to understand that in elderly fracture patients visiting an FLS, a DXA should be made. Thus, adding a VFA, or another imaging technique when VFA is not available, is fully in line with the IOF CtF programme and other programmes, and it can be a substantial step forward in optimizing patients care in osteoporosis.

The learning objectives of the minisymposium are:

- $\quad$ to recognize the clinical relevance of vertebral fractures;

- to be able to make a reliable radiological diagnosis of vertebral fractures with VFA;

- to understand the pros and cons of vertebral fracture assessment.

At the end of the mini-symposium, your questions are very welcome during the discussion.

\section{NSS59}

\section{CLINICAL SIGNIFICANCE OF VERTEBRAL} FRACTURES

J. Paccou $^{1}$

${ }^{1}$ University of Lille, CHU Lille, MABlab UR 4490, Department of Rheumatology, Lille, France

Previous studies have identified spine X-ray and vertebral fracture assessement (VFA) to detect prevalent vertebral fractures that improve prediction of future vertebral fractures and non-vertebral fractures. Moreover, more severe or greater number of vertebral fractures were associated with higher fracture risk than milder or fewer vertebral fractures. The risk of sustaining subsequent vertebral fractures is a phenomenon often referred to as "vertebral fracture cascade".

Vertebral fractures have many potential consequences for the individual beyond economic cost; these risks include functional limitation, loss of independence, pain, impaired quality of life, higher inpatient healthcare utilization and diminished lifespan. Health-related quality of life (HRQL) has become an important tool used to assess the burden of vertebral fractures. The severity and number of vertebral fractures are related to 
HRQL. Moreover, studies investigating time since onset of vertebral fracture, and its effect on HRQL, have shown that a residual effect lasts for up to 7 years post-fracture.

\section{NSS60 \\ RADIOLOGICAL DIAGNOSIS OF VERTEBRAL FRACTURES WITH VFA \\ J. Zhang ${ }^{1}$ \\ ${ }^{1}$ MRC, Lifecourse Epidemiology Unit, Southampton, Southampton, United Kingdom}

The burden of vertebral fractures (VF) is a global epidemic effecting between $30-50 \%$ of people over the age of 50 years. However, the accurate description of the epidemiology of vertebral fractures is complicated by a significant proportion that are clinically 'silent', and the existence of various definitions of vertebral fractures. Approaches to definition of vertebral fractures include morphological, morphometric, qualitative and quantitative.

Vertebral fracture assessment, VFA, has been proposed as an alternative approach for the identification of VF. The current conventional practice for initial assessment is the use of the spinal radiographs, which has remained as the (gold) standard that VFA images are compared to in studies establishing the validity, and reproducibility of VFA.

VFA is currently under-utilised globally and this can be due to a number of factors including its accuracy, cost and utility of the procedure been unclear to healthcare providers and payers. However, VFA has shown added value to clinical diagnosis of osteoporosis and its subsequent management. The use of VFA enhances the assessment especially for those in the normal and osteopenic BMD range, where, without the detection of VF through VFA, medication is unlikely to be commenced. VFA should be performed at the point of bone density measurement as part of a broader strategy to close the treatment gap.

NB I collaborated in this work with Nicholas Fuggle, Elaine Dennison, Cyrus Cooper

\section{NSS61}

\section{ADVANTAGES AND LIMITATIONS OF VERTEBRAL FRACTURE ASSESSMENT (VFA)} M. Chandran ${ }^{1,2}$

${ }^{1}$ Chairperson, Asia Pacific Consortium on Osteoporosis, Singapore, Singapore, ${ }^{2}$ Senior Consultant and Director, Osteoporosis and Bone Metabolism Unit, Department of Endocrinology, Singapore General Hospital, Singapore, Singapore

Vertebral fracture assessment (VFA) refers to lateral spine imaging done concurrently with axial bone densitometry. It has been shown to identify moderate to severe prevalent vertebral fractures accurately. Identification of prevalent vertebral fractures at the time of bone density measurement improves estimates of patients' risk of subsequent fractures and can help to identify appropriate candidates for pharmacological therapy. Other imaging modalities, including conventional Spine Xrays, MRI, CT, and nuclear bone scans, can identify vertebral fractures. CT and MRI provide improved resolution, a better picture of overall anatomy, the ability to evaluate the acuity of the fracture, and to differentiate between osteoporotic and non-osteoporotic (e.g. malignant) fracture. These modalities however require a separate visit and /or location to perform the study and result in significantly higher radiation exposure and/or cost. Another advantage of VFA is that the it provides a single image of both thoracic and lumbar spine and the images have less parallax distortion compared to conventional spine radiographs.

VFA however is associated with certain limitations. It cannot determine the acuity of the fracture nor can it elucidate its aetiology. It, as with Spine X-rays and other imaging modalities relies upon the experience of the technician performing the study and the expert interpreting it. The agreement between spine radiography and VFA for mild fractures (grade 1 ) is lower than that seen for more moderate or severe (grade 2 and 3) fractures, with VFA having lower sensitivity to detect milder fractures. The image quality and resolution are superior with conventional radiographs compared to VFA, particularly at the upper thoracic regions (above T6 or T7). Despite these limitations, VFA is an excellent imaging modality to evaluate for the presence of most vertebral fractures and should be considered if resources permit as part of routine axial densitometry.

\section{NSS62}

\section{TRENDS IN FRACTURE INCIDENCE IN HIGH-} INCOME COUNTRIES

B. Abrahamsen ${ }^{1,2,3}$

${ }^{1}$ Department of Clinical Research, Odense Patient Data Explorative Network, University of Southern Denmark, Odense, Denmark, ${ }^{2}$ Department of Medicine, Holbæk Hospital, Holbæk, Denmark, ${ }^{3}$ Nuffield Department of Orthopaedics, Rheumatology and Musculoskeletal Sciences, University of Oxford, Oxford, United Kingdom

Background: Epidemiological information allowing for calculation of fracture rates have been available at a nationwide level in many high-income countries since in 1980 s, while regional data collections have been taking place in some areas like Rochester Minnesota since 1928 and in the Malmo area of Sweden since 1950. The quality of the information is particularly strong for hip fractures due to the nature of the surgical intervention while the information on fractures that are treated in an outpatient setting can be prone to incomplete or duplicate recording at least in some healthcare systems. As will be discussed, the information on spine fractures is particularly 
uncertain due to a low diagnostic effort and suboptimal standards of reporting. The general expectation is that increased industrialisation and a longer lifespan in high income countries should be accompanied by an absolute increase in fracture burden, especially for fractures that are linked to a more sedentary working life.

Methods: Narrative review of published epidemiology studies for high-income countries.

Findings: For hip fractures, most but not all high-income countries saw a reversal of the increasing trend in both men and women during the past two decades, albeit with the inflection point differing by country. The age at hip fracture appears to be slightly increasing in the Nordic countries and the female:male ratio is beginning to decrease. It is unclear whether this is driven by period or cohort effects but studies for Sweden and Denmark suggest that younger birth cohorts will increase their hip fracture rates. In accordance with this, Swedish data suggest that forearm fractures may also be reverting to an increased risk, with the increase being particularly prominent for the 50-60 age group.

Conclusion: Despite a reassuring trend of diminishing hip fracture rates in high-income countries over the past two decades, the incidence of sentinel osteoporotic fractures such as forearm fractures show signs of potentially being on the rise in accordance with predictions that we may be approaching a reversal with a new increase in hip fracture rates based on APC models.

\section{NSS63}

BREAKING THE MYTH IN SUB-SAHARAN AFRICA - WHAT DO WE KNOW ABOUT FRAGILITY FRACTURE?

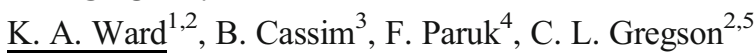

${ }^{1}$ MRC Lifecourse Epidemiology, Human Development and Health, University of Southampton, Southampton, United Kingdom, ${ }^{2}$ SAMRC Developmental Pathways for Health Research Unit, School of Clinical Medicine, University of the Witwatersrand, Johannesburg, South Africa, ${ }^{3}$ Department of Geriatric Medicine, School of Clinical Medicine, College of Health Science, University of KwaZulu Natal, Kwa-Zulu Natal, South Africa, ${ }^{4}$ Division of Internal Medicine, School of Clinical Medicine, College of Health Science, University of Kwa-Zulu Natal, Kwa-Zulu Natal, South Africa, ${ }^{5}$ Musculoskeletal Research Unit, Bristol Medical School, University of Bristol, Bristol, United Kingdom

Objective: The number of older adults (aged $\geq 60$ years) in Sub-Saharan Africa (SSA) is two times higher than in northern Europe and that number is rising exponentially. As SSA undergoes an epidemiological transition as a result of rapid urbanisation, the burden of non-communicable diseases, including osteoporosis, is increasing yet osteoporosis is often undiagnosed until a fragility fracture occurs ${ }^{1}$. The risk factors for fragility fractures in this context include high HIV prevalence, under- and over nutrition, high trauma rates and marked socioeconomic inequalities. The objective is to review the current and emerging evidence from African countries at differing stages of the epidemiological transition.

Methods: Narrative review of published epidemiology studies in SSA countries

Findings: There are limited data from SSA, with studies often retrospective, small and based in a single-hospital setting. The first study of hip fracture incidence in South Africa has been conducted in a multi -centre, multi-ethnic population ${ }^{2}$. Ethnic- and gender specific hip fracture rates will be reviewed in this symposium, together with the findings of 12-month mortality. The prevalence of vertebral fractures was assessed in an opportunistic sample in a small study from South Africa, showing similar frequencies in black $(9 \%)$ and white $(5 \%)$ women ${ }^{3}$. A similar vertebral fracture prevalence $(9 \%)$ was observed in The Gambian Bone Ageing Study of 488 Gambians aged $40+$ residing within 10 survey villages in rural Gambia; 3\% women, $0.4 \%$ men self-reported hip fracture/ fracture-like deformities and $10 \%$ women, $0.4 \%$ men an arm fracture $^{4}$.

Conclusion: Increasing evidence is now dispelling the outdated myth that fragility fractures are not a problem in SSA. There is limited evidence from across the regions, particularly in the most resource poor countries. There is an urgent need to act to determine the burden of fragility fractures, preparedness of healthcare systems and to identify risk factors for fracture. Only then can prioritisation for prevention of this rising burden begin.

\section{References}

1. Gregson et al. Lancet Glob Health. 2019 Jan;7(1):e26-e27

2. Dela et al. Bone 2020 (in press)

3. Conradie et al. Arch Osteoporos. 2015;10:203.

4. Zengin et al. Front Endocrinol (Lausanne). 2017 Aug 31;8:219.

\section{NSS64 \\ UPDATE ON MANAGEMENT OF LOW BONE MASS IN YOUNG WOMEN}

A. Orcesi Pedro ${ }^{1,2}$

${ }^{1}$ Professor of post-graduate course of Obstetrics and Gynecology - UNICAMP Medical School, Campinas, Brazil, ${ }^{2}$ President of National Committee on Osteoporosis of FEBRASGO, Campinas, Brazil

Although low bone mass and accelerated bone loss can occur early in life, osteoporosis is usually considered a disorder of postmenopausal women. To reduce the risk of osteoporosis, all women regardless age, should be encouraged to maintain a healthy lifestyle, regular physical activity and a balanced nutrition and knowledge about risk factors for bone loss. 
Gynecological perspectives to prevent osteoporosis consist in primary care during routine gynecological and obstetric assistance for women of all age groups. This presentation addresses the most relevant situations where adequate medical assistance may interfere positively on the purpose of preserving bone health during women's lifespan.

Certain groups of premenopausal women are at high risk of osteoporosis, including those with disease states, hormonal impairment and exogenous influences that promote accelerated bone loss. Osteoporosis prevention, diagnosis and therapy specified peak bone mass in children and secondary osteoporosis in young adults are important issue that needs to be addressed by Gynecologists, as a woman's primary physician. The diagnosis of osteoporosis should only be considered in premenopausal women with existing fragility fractures, diseases or treatments known to cause bone loss or fractures. Secondary causes of osteoporosis should be corrected or treated if possible. The women should be recommended sufficient intake of calcium and vitamin and physical activity. In women with recurrent fractures or secondary causes that cannot be eliminated, for example glucocorticoid or cancer treatment, pharmacological intervention with bisphosphonates or teriparatide may be considered.

\section{NSS65}

\section{BONE MASS AND FRACTURE RISK DURING PREGNANCY AND LACTATION}

B.-H. Albergaria ${ }^{1,2,3,4}$

${ }^{1}$ Professor of Clinical Epidemiology- Federal University of Espirito Santo, Vitória, Brazil, ${ }^{2}$ Vice-President of National Osteoporosis Commission - FEBRASGO, Vitória, Brazil, ${ }^{3}$ Secretary of the Brazilian Association of Bone Evaluation and Osteometabolism -ABRASSO, Vitória, Brazil, ${ }^{4}$ Technical Director -CEDOES Diagnosis and Research of Osteoporosis, Vitória, Brazil

Objectives: To summarize our current state of knowledge about the pathophysiology, incidence, and treatment of osteoporosis that presents during pregnancy, puerperium, and lactation.

Materials \& Methods: In this presentation, we review our current understanding of the pathophysiology of fragility fractures that occur for the first-time during pregnancy and lactation, and provide guidance on appropriate investigations and treatment strategies. Most affected women will have had no prior bone density reading, and so the extent of bone loss that may have occurred during pregnancy or lactation is uncertain. During pregnancy, intestinal calcium absorption doubles in order to meet the fetal demand for calcium, but if maternal intake of calcium is insufficient to meet the combined needs of the mother and baby, the maternal skeleton will undergo resorption during the third trimester. During lactation, several hormonal changes, independent of maternal calcium intake, program a 5-10\% loss of trabecular mineral content in order to provide calcium to milk. After weaning the baby, the maternal skeleton is normally restored to its prior mineral content and strength. This physiological bone resorption during reproduction does not normally cause fractures; instead, women who do fracture are more likely to have additional secondary causes of bone loss and fragility. Transient osteoporosis of the hip is a sporadic disorder localized to one or both femoral heads; it is not due to generalized skeletal resorption. Case reports have used anti osteoporotic drugs to treat pregnancyassociated osteoporosis; however, the need for such treatments is uncertain given that spontaneous skeletal recovery that normally occurs after pregnancy-weaning.

Results: These relatively rare fragility fractures result from multifactorial causes, including skeletal disorders that precede pregnancy, and structural and metabolic stresses that can compromise skeletal strength during pregnancy and lactation. Further study is needed to determine when pharmacological or surgical therapy is warranted instead of conservative or expectant management.

Conclusion: Pregnancy-associated osteoporosis is a rare syndrome affecting women during late pregnancy and the early postpartum period; notwithstanding, clinicians should be aware of this condition as a recognized complication of pregnancy. The condition should be especially considered in women presenting with new onset back pain in pregnancy or the postpartum period.

\section{NSS66}

EVALUATION AND MANAGEMENT OF OSTEOPENIA AND OSTEOPOROSIS IN BREAST AND GYNECOLOGICAL CANCER SURVIVORS

\section{L. Steiner ${ }^{1,2}$}

${ }^{1}$ Department of Obstetrics and Gynecology - ABC Foundation School of Medicine, Santo André, Brazil, ${ }^{2}$ Member of the National Committee on Osteoporosis of FEBRASGO, Santo André, Brazil

Cancer is the second cause of death among female in worldwide, with breast cancer being leading cause and accounting for $25 \%$ of cancer cases and $15 \%$ of cancerrelated deaths. The overall cancer death rate dropped continuously from 1991 to 2016 by a total of $27 \%$, translating into approximately 2,629,200 fewer cancer deaths than would have been expected if death rates had remained at their peak. New pharmacological approaches and treatment improvements are the cornerstone for this scenario, but they come with a price. Invariable, cancer-treatment strategies are harmful to bone and associated with increased risk for osteoporosis and fragile fractures. Coalescence of cancer survivors and osteoporosis is becoming a health problem of near-epidemic proportions. 
Cancer treatment-induced bone loss (CTIBL) are chemotherapy, radiotherapy, chemical or surgical castration, and hormone therapy. Bone loss that occurs with these therapies are more rapid and severe than postmenopausal bone loss in women and rates of bone loss can be more than seven-fold higher than in normal aging. The primary cause of CTIBL is estrogen decrease. Other mechanisms include direct or indirect effects of cancer therapies or malignancy on bone metabolism, inactivity, and inadequate intake of calcium and vitamin D. Also, there is a lack of awareness how cancer itself can impact negatively bone tissue, for example, RANKL increase is related to breast carcinogenesis and bone loss, suggesting that women with breast cancer may have loss bone mass.

Since CTIBL causes bone fragility and an increased susceptibility to fractures; prevention, early diagnosis, and treatment of CTIBL are essential to decrease the risk of fracture. Clinicians must recognize which patients with cancer are at risk and which should undergo screening and surveillance and at what frequency. Generally, in postmenopausal women diagnosed with cancer fracture, risk assessment and treatment intervention are based on bone mass density and clinical risk factors for osteoporosis. Some guidelines recommend pharmacological intervention with cutoff of T-score $<-2.0 \mathrm{sd}$, instead common $\mathrm{T}$-score of $<-2.5 \mathrm{sd}$. In contrast to postmenopausal women, there is no specific guideline to recognize premenopausal women with high risk to osteoporosis before initiate CTIBL use. Considering lower incidence of low bone mass and scarce evidence of benefits outweigh harms, almost none are screened before receive chemotherapy, GnRH analogs and tamoxifen. Personalized instead one-size-fits-all approach are mandatory in these cases.

Clinicians should actively encourage patients to follow lifestyle measures which benefit bone health. Pharmacological intervention is recommended in women with high risk for osteoporotic fracture. The current evidence suggests oral bisphosphonates, intravenous bisphosphonates, and subcutaneous denosumab are each efficacious option. The choice of which bone-modifying agent to offer should be based on several important considerations, including patient preference, potential adverse effects, quality-of-life considerations, adherence, safety for that population, cost, and availability.

\section{NSS67}

\section{SARCOPENIA. DIAGNOSIS AND EVALUATION}

P. Athanassiou ${ }^{1}$

${ }^{1}$ Department of Rheumatology, St. Paul's Hospital, Thessaloniki, Greece

Sarcopenia is defined as an age associated decline in skeletal muscle mass and muscle strength. The pathophysiology of sarcopenia is multifactorial, with decreased caloric intake, muscle fiber denervation, intracellular oxidative stress, hormonal decline, and enhanced myostatin signaling, which are all thought to contribute. Prevalence rates are high in elderly community dwelling and long-term care populations with advanced age, low body mass index, and low physical activity as significant risk factors. Sarcopenia shares many characteristics with other disease states typically associated with risk of fall and fracture, including osteoporosis, frailty, and obesity. Sarcopenia is a syndrome characterized by progressive and generalized loss of skeletal muscle mass and strength with a risk of adverse outcomes such as physical disability, poor quality of life and death. The presence of both low muscle mass and low muscle function (strength or performance) is required for the diagnosis of sarcopenia. Low muscle mass and low muscle strength or low physical performance are the criteria for the diagnosis of sarcopenia.

Diagnosing sarcopenia requires assessment of muscle mass, muscle strength, and physical performance. Muscle mass may be estimated by computed tomography (CT), magnetic resonance imaging (MRI), dual energy X-ray absorptiometry (DXA) or bioimpedance analysis (BIA). Muscle strength may be estimated by handgrip strength. Physical performance may be measured by the usual gait speed, the get-up-and-go test or the timed get-up-and-go test.

Screening for sarcopenia is recommended for elderly patients and those with conditions which reduce physical function. The diagnosis of sarcopenia will lead to the recognition of the problem and its proper management. Various agents are in the pipeline which will contribute to the treatment of sarcopenia and will lead to what is the aim in modern medicine, i.e. healthy aging.

\section{NSS68}

\section{SARCOPENIA AND NUTRITION}

L. Athanassiou $^{1}$

${ }^{1}$ Department of Physiology, Medical School, University of Athens, Athens, Greece

Healthy aging is the aim of modern medicine. The maintenance of independence and quality of life is crucial for the aging population, which increases dramatically. One of the threats to independent life is the loss of muscle mass and muscle function, which is called sarcopenia. Sarcopenia is one of the main factors which contribute to frailty. It can lead to functional impairment and mobility limitation.

Resistance training and adequate protein and energy intake are the key strategies for the management of sarcopenia. Management of weight loss and resistance training are the most relevant protective countermeasures to slow down the decline of muscle mass and muscle strength. The quality of amino acids in the diet is an important factor for stimulating protein synthesis. Additionally, it appears that the use of certain foods, such as milk, cheese and yoghurt may have 
beneficial effects on sarcopenia, more than the nutrients these foods contain. The use of dairy matrix is important for the management of sarcopenia, as it has been shown that certain foods may have more beneficial effects than their nutrient content. It is important to note that the nutritional content of certain food items may vary dramatically according to the geographical area of the patients' residence. Therefore, these factors should be borne in mind and taken care of. The daily amount of protein and dairy products should ideally be divided in the meals consumed over the day as it appears that the amino acid intake may be a stimulus for muscle formation.

In conclusion, adequate energy and protein intake and physical activity are the main anabolic stimuli for muscle protein synthesis and for the management of sarcopenia.

\section{NSS69}

\section{SARCOPENIC OBESITY. DEFINITION AND PATHOPHYSIOLOGY}

I. Kostoglou-Athanassiou $^{1}$

${ }^{1}$ Department of Endocrinology, Asclepeion Hospital, Voula, Athens, Greece

The simultaneous presence of sarcopenia and obesity is called sarcopenic obesity. Sarcopenic obesity has been defined as a distinct entity. It is characterized by a vicious cycle between muscle and fat tissue incorporating proinflammatory cytokines, oxidative stress and insulin resistance and may contribute to frailty and the risk of death.

A dramatic change associated with human aging is the progressive decline of skeletal muscle mass. Sarcopenia is an age-related loss of muscle mass and decline in muscle strength. Sarcopenia is defined as "appendicular skeletal mass divided by body height squared in meters (muscle mass index)" two standard deviations or more below reference values from young healthy individuals measured with dual X-ray absorptiometry. More recently criteria for sarcopenia have been proposed which are based on the amount of lean mass being lower than expected for a given amount of fat mass. Both muscle mass and muscle strength decline with aging but the decline in strength is greater than that expected based on the decline in mass. At the cellular level sarcopenia is accompanied by a loss of innervation and adaptive changes in the proportions of slow and fast motor units, as well as in the cross-sectional area of muscle fibers. Obesity is an important health threat that is a major risk factor for metabolic and cardiovascular morbidity and mortality. There is a world-wide increase in the prevalence of obesity in middle-aged and older adults. The major age-related changes in body composition include an increase in body fat and a decline is skeletal muscle.
Sarcopenic obesity refers to the simultaneous presence of sarcopenia and obesity. The complex interplay of common pathophysiological mechanisms such as increased proinflammatory cytokines, oxidative stress, insulin resistance, hormonal changes and decreased physical activity underlie the close relationship between sarcopenia and obesity. A vicious cycle may take place between the accumulation of fat and the loss of skeletal muscle mass since they have a reciprocal influence on each other. Sarcopenia reduces physical activity, which leads to decreased energy expenditure and increases the risk of obesity. An increase in visceral fat induces inflammation, which contributes to the development of sarcopenia. Both sarcopenia and obesity are associated with metabolic disorders, morbidity and mortality. Nowadays, exercise along with proper adaptation in nutrition to promote weight loss without accompanying further muscle loss may be used for the prevention and treatment of sarcopenic obesity.

It appears, that sarcopenic obesity is a common entity which may contribute to frailty and the risk of death.

\section{NSS70}

\section{SARCOPENIC OBESITY. MANAGEMENT}

Y. Dionyssiotis $^{1}$

${ }^{1}$ 1st Physical Medicine \& Rehabilitation Department, National Rehabilitation Center EKA-KAT, Athens, Greece

Sarcopenic obesity is a common entity. Inactivity and improper dietetic habits contribute to the increase in the prevalence of this entity in the population.

There are recommendations for sarcopenia and / or weight loss. Strength exercise combined with proper diet have demonstrated positive effects on muscle function and a combination of dietary weight loss intervention and additional protein supplements may reduce body fat. However, there is a lack of studies with interventions and their outcome to deal with sarcopenic obesity. Nutrition is a common factor in both entities: sarcopenia and obesity, although interventions differ due to different pathophysiological mechanisms causing the problem: inadequate nutrition vs. excess consumption is the case. The problem is how to increase muscle mass in a situation of energy deficit, while during weight loss, which is effective in reducing fat mass, skeletal muscle mass may also be lost and consequently reduced. Higher protein intake prevents muscle mass loss, especially when combined with an exercise intervention. Exercise programs containing strength and aerobic exercise in combination with a dietary weight loss program may possibly have positive effects on sarcopenic obesity.

It appears that the management of sarcopenic obesity requires a program of both exercise and proper diet interventions. 


\section{NSS71}

TOWARDS A UNIFORM LANGUAGE OF FRAILTY R. Vella Azzopardi ${ }^{1}$, S. Vermeiren ${ }^{1}$, E. Gorus ${ }^{1}$, A. Habbig ${ }^{1}$, M. Petrovic ${ }^{2}$, N. Van Den Noortgate ${ }^{2}$, P. De Vriendt ${ }^{1}$, I. Bautmans ${ }^{1}$, I. Beyer ${ }^{1}$. On behalf of the Gerontopole Brussels Study group. https://fria.research.vub.be/en/butterfly-study

${ }^{1}$ Frailty in Ageing Research department (FRIA), Vrije Universiteit Brussel (VUB), Brussels, Belgium, ${ }^{2}$ Department of Geriatrics, Ghent University Hospital and Ghent University, Ghent, Belgium

Objective: The objective is to assess how the available frailty instruments operationalize frailty in comparison to an internationally standardised framework of health and its positive (function) and negative consequences (disability) called the International Classification of Functioning, Disability and Health (ICF).

Material and methods: A systematic review on frailty instruments using the search terms ("Aged"[Mesh] OR "Frail Elderly"[Mesh] OR "Aged, 80 and over"[Mesh]) AND Frailty AND ("Diagnosis"[Mesh] OR "Risk Assessment"[Mesh] OR "Classification"[Mesh]) was carried out in PubMed, Web of Knowledge and PsycINFO. Using the established Cieza's ICF linking rules each item of the identified frailty instruments was linked to the most detailed ICF code.

Results: The literature search generated 1,984 potential articles. Sixty-seven articles were included in this systematic review. Seventy-nine original or adapted frailty instruments were identified and subdivided into single(physical $\mathrm{n}=24$; co-morbidities $\mathrm{n}=1$ ) and multi-domain $(n=54)$ categories. Only 5 frailty instruments (indexes) were linked to all 5 ICF components. The most frequently linked ICF components were Activities \& Participation $(\mathrm{n}=522)$ and Body Functions $(\mathrm{n}=507)$. Body Structures $(\mathrm{n}=55)$, Environmental $(\mathrm{n}=21)$ and Personal factors $(\mathrm{n}=64)$ were sparingly represented mainly in the multi-domain frailty instruments. 60 frailty items could not be linked to an ICF code. One hundred and twenty-four frailty items represented comorbidities and so were not given an ICF code but designated the term 'health condition'.

Conclusion: This review highlights 4 salient points. First, there is heterogeneity in current frailty operationalization. A common frailty language is crucial to facilitate clear communication and comparability in research as well as in clinical practice. Secondly, certain domains of frailty e.g. social factors which hinder or facilitate the individual's intrinsic frailty status (social frailty) and assessment of advanced activities of daily living which capture subtle decline in function associated with mild cognitive impairment (cognitive frailty) are underrepresented. Thirdly, environmental (e.g. relationships) and personal factors (e.g. gender) are depreciated despite evidence that they contribute to frailty. Finally, this review showed inconsistency among the frailty instruments regarding whether co-morbidity and frailty are separate entities. In conclusion, this systematic overview and ICF translation can be a cornerstone for future standardization of frailty assessment.

\section{NSS72}

\section{THE OPERATIONALIZATION OF FATIGUE IN FRAILTY SCALES}

V. Knoop ${ }^{1}$, A. Costenoble ${ }^{1}$, R. Vella Azzopardi ${ }^{1}$, S. Vermeiren $^{1}$, A. Debain ${ }^{1}$, B. Jansen ${ }^{2}$, A. Scafoglieri ${ }^{3}$, I. Bautmans ${ }^{1}$. On behalf of the Gerontopole Brussels Study group. https://fria.research.vub.be/en/butterfly-study

${ }^{1}$ Frailty in Ageing Research department (FRIA), Vrije Universiteit Brussel (VUB), Brussels, Belgium, ${ }^{2}$ Department of Electronics and Informatics (ETRO), Vrije Universiteit Brussel (VUB), Brussels, Belgium, ${ }^{3}$ Experimental Anatomy, Vrije Universiteit Brussel (VUB), Brussels, Belgium

Objectives: Fatigue is a common complaint among older adults and is a strong predictor for the onset of disabilities, loss of independence, mortality and other adverse health outcomes. Because of the common biomedical determinants for muscle fatigue and frailty and because of the established relationship of fatigue with the core elements of frailty, fatigue could be an important clinical feature in the early stages of frailty. This presentation aims to give an overview of the different fatigue items that are used in the existing frailty scales. Material and Methods: PubMed, Web of Knowledge and PsycINFO were systematically screened for frailty scales (last search on September 30th, 2018). 133 articles were included, describing 158 frailty scales. Fatigue items were extracted and categorized in 4 fatigue constructs: "mood state related tiredness", "general feeling of tiredness", "activity based feeling of tiredness" and "resistance to physical tiredness".

Results: 120 fatigue items were identified, of which 100 belonged to the construct "general feeling of tiredness" and only 9 to the construct "resistance to physical tiredness". $49,4 \%$ of the frailty scales included at least 1 fatigue item, representing $15 \pm 9,3 \%$ of all items in these scales. Fatigue items have a significantly higher weight in single domain (dominantly physical frailty scales) versus multi domain frailty scales $(21 \pm 3.2$ versus $10.6 \pm 9.8 \%, p=<0,05)$.

Conclusion: Fatigue is prominently represented in frailty scales, covering a great diversity in fatigue constructs and underlying pathophysiological mechanisms by which fatigue relates to frailty. Although fatigue items were more prevalent and had a higher weight in physical frailty scales, the operationalization of fatigue leaned more towards psychological constructs. 


\section{NSS73}

\section{THE DISABILITY PARADOX IN THE FRAILTY CONSTRUCTS}

A. Costenoble $^{1}$, V. Knoop ${ }^{1}$, A. Debain ${ }^{1}$, S. Vermeiren ${ }^{1}$, R. Vella Azzopardi ${ }^{1}$, G. Rossi ${ }^{2}$, C. Smeys ${ }^{1}$, K. Duarte Baltazar ${ }^{1}$, I. Bautmans ${ }^{1}$, D. Verté ${ }^{3}$, E. Gorus ${ }^{1}$, P. De Vriendt ${ }^{1}$. On behalf of the Gerontopole Brussels Study group. https://fria.research. vub.be/en/butterfly-study

${ }^{1}$ Frailty in Ageing Research department (FRIA), Vrije Universiteit Brussel (VUB), Brussels, Belgium, ${ }^{2}$ Personality and Psychopathology Research Group, Vrije Universiteit Brussel (VUB), Brussels, Belgium, ${ }^{3}$ Belgian Ageing Studies research group, Vrije Universiteit Brussel (VUB), Brussels, Belgium

Background and Objectives: The relationship between frailty and disability in activities of daily living (ADLs) can be seen in different ways, with disability being - to varying degrees - a characteristic, negative outcome, or predictor of frailty. This conflation of definitions is partly a result of the different frailty tools used in research. Aiming to provide a comprehensive overview, a systematic literature search analyzed (i) if, (ii) to what extent, and (iii) how ADLs are evaluated by frailty instruments.

Research Design and Methods: A search was performed in PubMed, Web of Knowledge, and PsycINFO to identify all frailty instruments, followed by categorization of the ADL items into basic (b-), instrumental (i-), and advanced (a-) ADLs. Results: In total, 192 articles described 217 frailty instruments, from which $52.1 \%$ contained ADL items: $45.2 \%$ bADLs, $35.0 \%$ i-ADLs, and $10.1 \%$ a-ADLs. The most commonly included ADL items were bathing (b-ADLs); using transportation (i-ADLs); and semi-professional work engagement in organized social life or leisure activities (a-ADLs). These instruments all had a multidomain origin $\left(\chi^{2}=122.4\right.$, $\mathrm{p}<0.001$ ).

Discussion and Implications: Because $52.1 \%$ of all instruments included ADL items, the concepts of frailty and disability appear to be highly entangled. This might lead to circular reasoning, serious concerns regarding contamination, and invalid research results.

\section{NSS74}

\section{MEASURES OF COGNITION IN THE FRAILTY CONSTRUCTS}

M. Petrovic ${ }^{1}$, R. Vella Azzopardi ${ }^{2}$, S. Vermeiren ${ }^{2}$, I. Beyer ${ }^{2}$, N. Van Den Noortgate ${ }^{1}$, I. Bautmans ${ }^{2}$, E. Gorus ${ }^{1}$. On behalf of the Gerontopole Brussels Study group. https://fria.research. vub.be/en/butterfly-study

${ }^{1}$ Department of Geriatrics, Ghent University Hospital and Ghent University, Ghent, Belgium, ${ }^{2}$ Frailty in Ageing Research department (FRIA), Vrije Universiteit Brussel (VUB), Brussels, Belgium
Objective: Advancing age is associated with both physical frailty and cognitive decline. The aim of this systematic review is to make an inventory of the inclusion and the operationalization of cognition in frailty instruments.

Material and methods: Pubmed, Web of Knowledge and PsychINFO were screened systematically until December 2016 using the keywords aged; frail elderly; aged, 80 and over; frailty; diagnosis; risk assessment and classification.

Results: Seventy-nine relevant articles encompassing 94 frailty instruments were included. Two instruments were excluded because they were insufficiently described. About half (46\%) of the identified frailty instruments included cognition. Of these, $85 \%$ were published after 2010 , with a significant difference for publication date $(\mathrm{X} 2=8.45, \mathrm{p}<.05)$, indicating a shift in the weight of cognitive measures in more recent frailty instruments. Seven methods of cognitive assessment were identified: dementia as co-morbidity; objective cognitivescreening instruments; self-reported; specific signs and symptoms; delirium/clouding of consciousness; non-specific cognitive terms and mixed assessments.

Conclusions: Although the concept of cognitive frailty has increasingly emerged in recent frailty instruments, it has been operationalized in many different ways. Future studies should focus on the identification of the cognitive domains affected in cognitive frailty, followed by the standardization of the operationalization of cognitive frailty. This could constitute the groundwork for the development of preventive interventional strategies for late-life functional decline.

\section{NSS75}

\section{ACUTE AND CHRONIC PAIN: THE DIFFERENCE BETWEEN DIAGNOSTIC APPROACHES}

O. Kurushina $^{1}$

${ }^{1}$ Volgograd State Medical University, Department of Neurology, neurosurgery, medical genetics, Volgograd, Russia

Pain is a complex experience that cannot be unambiguously perceived by the patient and easily diagnosed by the doctor. The variety of clinical forms of pain makes us look for additional approaches to the diagnosis of this syndrome. The report will consider various types of pain syndromes and will discuss various diagnostic methods that are most suitable for each specific type of algic disorder. The proposed diagnostic algorithms may be useful to doctors of various specialties in the management of patients with different diseases.

\section{NSS76}

\section{PAIN AND BIOMECHANICS}

A. Barulin ${ }^{1}$

${ }^{1}$ Volgograd State Medical University, Department of Neurology, neurosurgery, medical genetics, Volgograd, Russia 
Chronic pain syndromes have a multimodal origin, which is based on morphofunctional interactions, emotional, behavioral and social aspects. When creating an algorithm for diagnosing chronic pain syndromes, it is necessary to study the state of the body in a complex. Special role in this report is given to a little-studied topic - biomechanics and the interaction of psychoemotional and social aspects on it. Formation of cascade of biomechanical changes is discussed: dysregulation of muscle tone, visceral abnormalities in the spinal segment, dystrophic spinal disorders.

Especially important for patients with high risks (heavy physical work, static work postures and another).

\section{NSS77}

\section{FEATURES OF CLINICAL DIAGNOSIS OF CERVICOGENIC HEADACHE}

A. Drushlyakova $^{1}$

${ }^{1}$ Volgograd State Medical University, Department of Neurology, neurosurgery, medical genetics, Volgograd, Russia

The prevalence of cervicogenic headache in the population is as high as $14-20 \%$. Pathological changes in the upper three cervical spinal segments form the basis of the neuroanatomy of CGH. Diagnosis is based on the criteria of the International Classification of Headache Disorders. But it is challenging to diagnose clinically and must include the neurological, vertebroneurological and manual examination. The most common risk factors for the development of CGH are lasting static load, forced posture, a sedentary lifestyle, obesity, chronic stress. A violation of the musculoskeletal system which leads to abnormalities in the cervix increase in patients with risk factors.

\section{NSS78 \\ DIAGNOSIS OF TEMPOROMANDIBULAR DISORDERS IN PATIENTS WITH CHRONIC FACIAL PAIN \\ B. Kalinchenko ${ }^{1}$ \\ ${ }^{1}$ Volgograd State Medical University, Department of Neurology, neurosurgery, medical genetics, Volgograd, Russia}

Pain related to temporomandibular disorders (TMD) is a common problem in modern societies. The aim of the report is to present the modern methods for diagnosing TMD in patients with chronic pain. The effectiveness and possibility of using the developed methods of visual diagnostics in neurological patients with chronic myofascial pain syndrome will be presented. The report will demonstrate the possibilities of using this technique for myofascial facial pain. The report will consider the possibilities of conservative treatment including exercises, occlusal splint therapy, massage, manual therapy and others as the primary treatment for TMD pain.

\section{NSS79}

THE AWARENESS AND KNOWLEDGE OF OSTEOPOROSIS AND RISK FACTORS IN A GROUP TURKISH GERIATRIC PATIENTS

B. Karaoglan $^{1}$, M. Zinniroğlu ${ }^{1}$, P. Borman ${ }^{1}$

${ }^{1}$ University of Gazi Faculty of Medicine Dept of PMR, Ankara, Turkey

Objectives: Awareness about osteoporosis is still a concern in geriatric patients. The aim of this study was to evaluate the awareness and knowledge about osteoporosis and risk factors in a group of Turkish elderly.

Materails and Methods: A survey was conducted and performed by the members of 'Living with Osteoporosis' Foundation, in hospital settings, between 2018 October and 2019 April. The participants aged 60 and over were asked to answer a 10-minute survey, including 23 questions. In addition to their demographical properties, the questionnaire elicited data on knowledge and awareness about osteoporosis and risk factors. Results: Four hundred and twenty seven female and 44 male elderly completed the survey. The mean age of the subjects was $65.2+4.6$ years. $71 \%$ of the participants were aged between $60-$ 70 years. $68 \%$ of the elderly was graduated from lycee, $13 \%$ from university and $20 \%$ from primary school. $69 \%$ of individuals declared that they heard about osteoporosis however only $19 \%$ were aware of the risk factors. There was a positive correlation between knowledge about osteoporosis and education level of participants. $15 \%$ of the patients had osteoporosis and the presence of osteoporisis was related with family history and clothing style. Majority of the osteoporotic patients were female and using biphosphanate and VitD+calcium therapy.

Conclusion: Although awareness about osteoporosis is relatively high, elderly subjects had not enough knowledge about risk factors. In conclusion educational activities are needed for information on risk factors of osteoporosis and preventional methods in elderly subjects in order to increase quality of life.

\section{NSS80}

THE KNOWLEDGE ABOUT OSTEOPOROSIS IN A GROUP OF TURKISH FEMALES: RELATIONSHIP WITH QUANTITATIVE ULTRASONOGRAPHIC SCORES

P. Borman $^{1}$, B. Karaoglan ${ }^{2}$, B. D. Çakit ${ }^{3}$, G. T. Öztürk ${ }^{4}$, Z. Günendi $^{2}$

${ }^{1}$ University of Hacettepe Faculty of Medicine Dept of PMR, Ankara, Turkey, ${ }^{2}$ University of Gazi Faculty of Medicine Dept of PMR, Ankara, Turkey, ${ }^{3}$ Ankara Training and Research Hospital Clinic of PMR, Ankara, Turkey, ${ }^{4}$ Ankara PMR Training and Research Hospital Clinic of PMR, Ankara, Turkey

Objectives: Osteoporosis has become a major medical problem and is associated with an increased risk of fracture. 
Awareness and education about ostoporosis is low among the population especially in developing countries. The purpose of this cross-sectional study was to evaluate the knowledge in osteoporosis, symptoms, risk factors, treatment effects and to screen osteoporosis in a group of Turkish female subjects.

Materials \&Methods: Eligible candidates who attended to the educational meeting of ostoporosis on World Osteoporosis Day (20th October 2019) were recruited to the study. Demographic data regarding educational level,occupation, menopausal status, body mass index (BMI), fracture history, smoking, exercise and clothing style was recorded. Facts on osteoporosis quiz (FOOQ) detecting the knowledge about op, symptoms, risk factors and treatment was performed and quantitative ultrasonographic (QUS) densitometry from calceneus was evaluated before the educational program. Descriptive statistics were used for clinical variables and frequency of applied interventions. The females were graded as normal, osteopenic, and osteoporotic based on the QUS scores obtained.

Results: 68 peri/postmenopausal females with a mean age of $53.58 \pm 9.83$ years were recruited. Majority of the patients were married, housewies, having more than 1 child, and postmenopausal with a mean BMI of $29.76 \pm 4.44 \mathrm{~kg} / \mathrm{m}^{2} .8 .8 \%$ had a history of steroid prescription, $19 \%$ were smoking, $2.8 \%$ were on regular exercise (walking) and $81 \%$ had islamic clothing style. $19.1 \%$ had previous low energy fracture (mostly Colles fx). Of those nobody were assessed for op. The QUS scan revealed $33.8 \%$ were osteopenic and $20.6 \%$ were osteoporotic. No female had used any anti-osteoporotic drug. The most common belief was the increasement of fracture risk by presence of osteoporosis. The attendants had little knowledge about the symptoms and risk factors of osteoporosis and duration between menopausal status and osteoporosis. There was no association between QUS scores, and demographical properties and FOOQ scores of the subjects.

Conclusions: In conclusion knowledge on osteoporosis and risk factors is not sufficient among Turkish females. Osteoporosis may be more common than estimated in postmenoapusal females but assessment and and treatment rates are low despite the presence of low-energy fracture history. Screening for ostoporosis, early diagnosis and treatment are of importance. Therefore, increasing awareness among doctorsand educational activities for population are suggested to reduce the burden of this global epidemic condition.

\section{NSS81}

\section{LABORATORY OVERVIEW OF BONE TURNOVER MARKERS}

A. Sepici Dinçel $^{1}$

${ }^{1}$ Department of Medical Biochemistry, Faculty of Medicine, University of Gazi, Ankara, Turkey

The measurement of biochemical markers of bone turnover may reflect either enzymatic activities characteristic of the bone-forming or resorbing cells or bone matrix components released into circulation during resorption. None of the currently available bone markers have shown to be advantageous over others with regard to their clinical utility. In our different studies, we mainly focused on patient groups of osteoporotic hip fractures and their serum biochemical markers in order to discuss the risk assessments. A commonly used method to predict fracture risk is the FRAX tool developed by World Health Organization (WHO) since 2008 the method was released, no biomarker information has been incorporated into the prediction model. This tool and questions does not include any biochemical markers. The FRACTURK study was completed and estimated the current and future hip fracture risks and the prevalence of osteoporosis in Turkey. Besides FRAXbased intervention thresholds in men and women from Turkey were determined for our population. The use of biochemical biomarkers to diagnose and to monitor treatment effectiveness for early/silent states shall have economical and healthcare benefits. To be correlated the clinical state and biomarkers will help us to improve our expectations for the patients. Throughout our studies, along with common bone turnover markers, cytokines, trace elements, Vit D, antioxidants/oxidants, bone morphogenetic proteins, tryptophan and kynurenine pathway markers were evaluated for osteoporotic hip fractures. Biochemical markers were discussed within the fracture groups as intertrochanteric fractures or collum femoris fractures. And nowadays there has been a new approach which bases on our understanding of bone physiology. Related with that cathepsin-K, periostin, dickkopf-, sclerostin, sfingozine-1-phosphate and microRNAs can be considered as new biomarkers. Our fractured group studies had been included these new markers too. Amoung them local inhibitor factors of bone formation behaviour has been followed (patent pending TR/2019/20272). The clinical use of those biochemical markers are not fully established, their relationship with fracture risk has still have question marks and their use as treatment monitoring tools needs to be studied.

All those new mentioned markers can tell us about the osteocyte activities and distinguish the bone compartments that they might be helpful for exploring the pathological and physiological links between the bone, bone healing and other organs. Discovery of reliable biomarkers and development of efficient risk-predicting methodology are in great need to prevent the occurrence of osteoporotic fractures.

\section{NSS82}

RISK FACTORS OF OSTEOPOROSIS AND OSTEOPOROTIC FRACTURES

V. Sepici $^{1}$

${ }^{1}$ Emeritus Professor of Physical Medicine and Rehabilitation, Ankara, Turkey

All over the world osteoporosis is known as a common metabolic bone disease which is characterized by a decrease in 
bone mass and deterioration of bone microarchitecture. The most important complication of osteoporosis is fragility fractures. These fractures, which are usually seen in the elderly, are the most important complications that cause morbidity and mortality while putting a great burden to the economy. Peak bone mass in youth and bone loss rate at the beginning of bone loss can determine the risk of progressing osteoporosis. While increasing the awareness of osteoporosis especially in women, preventive studies should be worked, preventive training should be given and by surveillance studies risk groups should be identified. One of the important goals in the management of osteoporosis is to determine the probability of fracture risk. Briefly the risk factors in osteoporosis are; geriatric age, female gender, genetic factors, early menopause calcium and vit $\mathrm{D}$ deficiencies related with poor diet, insufficient sunlight, smoking, excessive consumption of alcohol, coffee, and fizzy beverage, overuse of sodium, sedentary lifestyle, long-term immobilization, nulliparous or multiparous, long-term use of corticosteroids, antiepileptic and antacid drugs and chemotherapy. In addition, endocrinologic disorders, inflammatory bowel disease, malabsorption, multiple myeloma, diseases of the central nervous system, and chronic inflammatory rheumatic diseases may increase the risk of osteoporosis. Although the risk factors of osteoporotic fractures are also eligible for the risk factors of osteoporosis, there can be additional risk factors like; low body mass index, sarcopenia, hip geometry, osteoporotic hip fracture history in family, impared vision and balance and neuromuscular disease, for fracturess. Apart from all these environmental factors and drugs should also be considered. In conclusion complete evaluation and preventive rehabilitation approaches are needed in order to desrease the risk of osteoporosis and osteoporotic fractures.

\section{NSS83}

\section{URIC ACID - FRIEND OR FOE?}

M. Bojinca ${ }^{1}$

1"Carol Davila" University of Medicine and Pharmacy, "Dr Ion Cantacuzino" Clinical Hospital, Bucharest, Bucharest, Romania

Uric acid is the final step of nucleic acids metabolism only for humans and great apes, because the rest of the mammals are able to produce uricase (urate oxidase), an enzyme that metabolizes uric acid to allantoin, which in turn, is highly soluble so easily excretable through urine. This situation is the consequence of a silencing mutation of uricase gene during evolution. As a result of it, the average serum level of uric acid is 3 to 5 times higher in humans and great apes in comparison with other mammals. Uric acid has antioxidant properties, it is maintaining blood pressure and is augmenting the capacity of fructose to induce fat stores, and all these features were providing survival advantages during evolution. However, increased level of uric acid (more than $7 \mathrm{mg} / \mathrm{dl}$ ) is associated with gout, metabolic syndrome and cardiovascular conditions, especially in developed countries. Uric acid seems to have some neuroprotective effects in Parkinson disease and Alzheimer. Humans and uric acid have a long common history and we need more data to decide if uric acid is a friend or a foe for the 21th century human being.

\section{NSS84}

\section{ASYMPTOMATIC HYPERURICEMIA - THE HIDDEN REALM (IMAGING CLUES)}

F. A. Vreju ${ }^{1}$

${ }^{1}$ University of Medicine and Pharmacy, County Clinical Hospital, Craiova, Craiova, Romania

Musculoskeletal ultrasound imaging method represents a major step forward on the journey to expose the intimate structure of this system and the pathophysiology of rheumatic diseases. One of the major advantages of using musculoskeletal ultrasound (MSK-US) is the possibility to discover abnormalities before they become clinically significant, thus symptomatic. That is, actually detection of pre-clinical disease. And this is the major target in rheumatology, as the early diagnosis is key to maintaining structure and function of the joints. In gout, there are some specific findings that intensely suggest the diagnosis. Besides ultrasound examination, in a person with hyperuricemia, the use of another developing imaging technique, the dual energy computed tomography (DECT), can lead to visualization of some abnormalities that may represent the consequence of urate deposits in the musculoskeletal system. Having said that, why could we not define, on the basis of imaging only, a new entity, pre-clinical gout, that we might as well call asymptomatic gout?! And this, might be the answer to the definition of therapeutic window of opportunity in gout, also.

\section{NSS85}

BONE MINERAL DENSITY IN HYPERURICEMIA AND GOUT - COLLATERAL DAMAGE?

C. Avram ${ }^{1}$, H. Popoviciu ${ }^{2}$

${ }^{1}$ West University of Timisoara, Timisoara, Romania,

2"George Emil Palade" University of Medicine, Pharmacy, Science and Technology of Targu Mures, County Clinical Hospital Targu Mures, Targu Mures, Romania

Uric acid (UA) is related to bone health through its antioxidant properties, or through the vitamin D-parathyroid hormone axis. Serum UA accounts for about $50 \%$ of extracellular antioxidant activity, suggesting that hyperuricemia may have a protective role in osteoporosis - disease characterized by high levels of oxidative stress.

Oxidative stress has a potential to attenuate osteoclastogenesis and bone formation. Whereas, antioxidants reduce osteoclastic activity, decrease bone resorption and increase 
differentiation of osteoblasts and mineralization process. Elderly osteoporotic women have lower antioxidant defenses as compared to normal age-matched population. When UA exists at supersaturated concentrations, such as in gout, its antioxidant properties could be overcome by its prooxidant effects, thus creating an environment of high oxidative stress despite the presence of high UA levels in the serum. The prooxidant role of UA may contribute to increased levels of circulating proinflammatory cytokines.

Clinical studies indicate controversial associations between the serum UA level and the bone mineral density (BMD), depending on gender, age, and the place of measurement of bone mineral density. A recent study demonstrated a significant positive association between high serum UA level and BMD at both femoral neck and spine in elderly men and women. Furthermore, UA levels between 4 to $4.99 \mathrm{mg} / \mathrm{dL}$ decrease the risk of osteoporosis in the elderly people aged 60 years and more.

Despite the protective effect of high serum UA on BMD, clinical implication of high serum UA in the elderly subjects remains to be determined, because, both high and low levels of UA are associated with metabolic, cardiovascular, renal and neurological diseases.

\section{NSS86 \\ GOUT TREATMENT - AS EASY AS IT SEEMS? \\ R. A. Ionescu ${ }^{1}$ \\ "Carol Davila" University of Medicine and Pharmacy, Colentina Clinical Hospital, Bucharest, Bucharest, Romania}

Gout treatment involves two elements: treatment of the gouty arthritis (attack) and treatment of hyperuricemia per se - urate lowering therapy (ULT); the scope of the latter is to restore the normal level of serum uric acid (sUA).

When treating the attack, there are three options from which to choose: colchicine, non-steroidal anti inflammatory drugs, and cortisone. When choosing between them we take into account the efficacy and safety of each of them, as well as the fact that colchicine is still as effective as when it was first used, one hundred years ago.

The ULT is also base on three therapeutic drug classes: inhibitors of uric acid synthesis, promotors of uric acid excretion (uricosurics) and synthetic uricase. When choosing between them, we have to questions to answer to: how do we choose the class and when do we initiate the treatment. To this day, the most used class of ULTs is xanthine oxidase inhibitors. There are two drugs that are part of this class: allopurinol, which is well known and widely used and febuxostat which, lately, seems to not be the "wonder drug" that it seemed to be in the beginning. Uricosurics are difficult to use drugs because of their complex posology but mostly because of the numerous adverse reactions they can elicit. In this class of drugs, lesinurad might "make a career".

\section{NSS87}

\section{ANGIOGENESIS AND BONE}

G. Holzer ${ }^{1}$

${ }^{1}$ Department of Orthopedics and Trauma Surgery, Medical University of Vienna. Vienna General Hospital, Vienna, Austria

Until recently vasculature of bone has not been in the focus of research, but accumulating evidence has shown that bone is a highly vascularized tissue. Vasculature plays an important role in physiological processes, like osteogenesis, and the dysregulation of vasculature is associated with many bone diseases such as osteoporosis or osteoarthritis. Vascular endothelial growth factor-A (VEGF) is one of the most important growth factors for regulation of vascular development and angiogenesis. VEGF influences skeletal development and postnatal bone repair and bone regeneration, but also regulates bone remodeling. Osteoblasts not only respond to VEGF stimulation, but also express and synthesize it. Normal and pathological osteoblasts produce and express VEGF. 1,25 dihydroxy-vitamin D3 treatment increases protein and $\mathrm{m}$ RNA VEGF levels. Pathological osteoblasts induce a strong angiogenic response, greater than observed of normal cells, suggesting the involvement of osteoblast-derived VEGF in the pathogenesis of bone diseases.

Studies of the functions of osteoblast-derived VEGF in healing of a bone defect indicate that osteoblast-derived VEGF plays critical roles at several stages of fracture healing and bone repair

Exercise or mechanical loading regulates bone vascularization in bone microenvironment via the modulation of angiogenic mediators (angiogenic and osteogenic coupling). The cross talk between osteoblasts and endothelial cells plays a pathophysiologic role in the response of the endothelium to unloading during disuse osteoporosis. This explains the underlying mechanisms of exercise and its role for osteoporosis prevention.

\section{NSS88}

\section{VASCULAR PATHOLOGY AND OA. IMAGING OF MICROCIRCULATION}

\section{J. J. Scali ${ }^{1}$}

${ }^{1}$ Head of Academic Unit of Rheumatology, Autoimmunity and Osteology- Durand University General Hospital of Buenos Aires, Buenos Aires, Argentina

Continuous growing evidence is stipulating a role to vascular pathology both in earliest stages and in OA progression. Besides, the crosstalk between endothelium, cartilage, and subchondral bone is probably another central feature in this process. The reduction of interstitial blood flow through the small vessels in the subchondral bone at the ends of long bones due to venous occlusion 
and stasis or presence of microemboli in subchondral bone vessels compromise the nutrient delivery and gas exchange in cartilage. This becomes a possibly initiator of cartilage breakdown in OA. Osteocyte apoptosis in subchondral bone would start osteoclastic bone resorption reducing almost temporarily, the bone support to the cartilage. Since the cartilage is avascular tissue, evidence accumulated shows that vascular problems may underlying the OA development. On the contrary, bone is a high vascular structure. The vasculature is involved in bone growth, repair and metabolism. The blood supply serves the bone marrow and calcified bone tissue, both tissues functionally interdependent regarding to hematopoesis, bone modelling and remodelling. Blood flow responsible for oxygen and nutrients exchange, metabolic waste with the interstitial fluid becoming very important for osteocytes located in the bone matrix, although osteoclasts and osteoblasts play a clear role in remodeling. Besides, osteocytes are the most numerous cells in bone and its adaptation to loading forces is clear, giving to these cells a role in bone mechanical sensing mediated by interstitial flow along osteocyte lacunae, driven by both mechanical loading of bone and pulsatile blood flow. Pathophysiology of OA is the venous stasis resulting in intraosseous hypertension and hypoxia. In response, osteoblast (OB) change their cytokine expression by accelerating bone remodeling and cartilage breakdown in OA. Animal models characterized the circulatory kinetics in OA bone with dynamic contrast enhanced MRI (DCE-MRI) and 18 F-PET studies demonstrated venous stasis and reduced perfusion. OB uptake of $18 \mathrm{~F}$ is consistent with the abnormal perfusion, bone remodeling and severity of OA. Circulatory kinetics with DCE-MRI in human knee OA exhibit similar venous outflow obstruction. Venous stasis is associated with hypoxia in subchondral bone. In OA exposed to hypoxia in vitro, there is upregulation of fibrinolytic peptides and deficiency in the regulation of PAI-1, leading to the generation of plasmin by human OA osteoblasts. Plasmin is a serine protease that has been shown to degrade cartilage in OA. Abnormal circulatory kinetics by DCE-MRI may be an image biomarker of OA. Pharmacologic modulation of venous stasis would have a positive venous effect on the physicochemical microcirculation of subchondral OB. The significant molecular interplay between subchondral bone vasculature and cartilage need further investigation particularly when increasing vascularity in subchondral bone is associated with OA severity. Regarding imaging of the microvasculature UTE (ultrashort echo time) MRI sequences with TEs particularly 100 times or less than the conventional clinical gradient echo sequences, can detect signal from cortical bones. DCE in cortical bone, perfusion parameters for cortical bone would be feasible. The relationship between bone perfusion, bone remodelling and fracture repair, increased bone turnover and inflammation is associated with increased blood flow. There is also a strong correlation between bone perfusion and bone mineral density (BMD). It is difficult to investigate perfusion with MRI because MRI in most soft tissue studies is not effective when applied to bone. UTE type sequences directly evaluate perfusion in cortical bone by reducing TEs from the several milliseconds of conventional sequences down to milliseconds for 2UTE sequences. Cortical bone perfusion can also be assessed with 18F-fluoride positron emission tomography (PET). However, 18F-Fluoride PET has a low spatial resolution and subjects exposed to ionizing radiation. MRI has an advantage because it can provide much higher spatial resolution without ionizing radiation.

\section{NSS89}

\section{THE MICROCIRCULATION ABNORMALITIES IN OSTEOPOROSIS AND OSTEOARTHRITIS}

\section{S. Sokolovic $^{1}$}

${ }^{1}$ University Clinical Center and Medical Faculty of Sarajevo Clinic for heart, blood vessels and rheumatic diseases, Sarajevo, Bosnia-Herzegovina

Background: The microcirculation and endothelial dysfunction play significant role in osteoporosis and osteoarthritis. Since the bone blood vessels are comprised only from endothelium, it is means that endothel pathology influence and trigger humoral cascade between osteoblasts and osteoclasts in order to enhance bone remodeling.

The failure of microcirculation in bone blood supply leads to osteopenia and osteoporosis.

Objectives: To investigate the scope of microcirculation abnormalitities in osteoporosis and osteoarthritis.

Methods: The open prospective parallel group study was designed. The following parameters will be observed. The coronary calcium score, arterial stiffness, the central and peripheral blood pressure, cardial output, augmentation index, peripheral resistance, mean arterial pressure, pulse pressure, cardiovascular risk factors, capillaroscopy, osteoporosis and osteoarthritis severity.

Result: The microcirculation and vascular abnormalaties have the great importance in a pathogenesis of osteoporosis and osteoarthritis. The arterial stiffness and rarefication of the capillaries has been detected in these degenerative rheumatic diseases. Vascular age, coronary calcium score is more prominent in osteoporosis and osteoarthritis compared to healthy controls. This is more significant when cardiovascular risk factors are applied.

Keywords: Microcirculation, Arterial stiffness, Osteoporosis, Osteoarthritis 


\section{NSS90}

NON-PHARMACOLOGICAL APPROACHES IN THE MANAGEMENT OF OSTEOPOROSIS. HOW TO MODIFY LIFESTYLE?

Y. Gokce Kutsal $^{1}$

${ }^{1}$ Hacettepe University Faculty of Medicine Dept of PMR, Ankara, Turkey

Non-pharmacological measures for osteoporosis prevention and treatment starts with the intervention and elimination of modifiable risk factors, and lifestyle and dietary measures should be taken into consideration seriously.

Calcium: The recommended calcium intake is 1000 and $1200 \mathrm{mg}$ daily, preferably through nutritional intake. Use dietary supplements only when calcium assumption is insufficient. In postmenopausal women and older men receiving bone protective therapy for osteoporosis, Ca supplementation should be given if the dietary intake is below $700 \mathrm{mg} /$ day. It is not recommended to exceed 500-600mg per dose. Supplementation is not recommended in older, noninstitutionalized adults.

Vitamin D: Sources of Vit D in foods are extremely limited. Although the main source of Vit D is sunlight exposure (UVB), it is not possible to achieve adequate Vit D levels exclusively from sunlight.Vit D supplementation considered in patients at risk of, or showing evidence of, Vit D insufficiency. In postmenopausal women and older men ( $\geq 50$ years) at increased risk of fracture a daily dose of 800 IU cholecalciferol should be advised. The objective is to reach a circulating concentration of $25(\mathrm{OH}) \mathrm{D}$ of between 30 and $50 \mathrm{ng} / \mathrm{mL}$ $(75-125 \mathrm{nmol} / \mathrm{L})$, that is stable over time. In elderly and the chronically ill, doses between 800 and 2000 IU are recommended (Safe upper level for Vit D is $4000 \mathrm{IU} /$ day).

Concerns: High doses of Vit D, particularly with calcium supplementation, can cause hypercalcemia, hypercalciuria, and kidney stones. Vascular calcification may contribute to the cardiovascular events observed in calcium supplement users. Protein: Sufficient dietary protein, ideally achieved through dairy products in postmenopausal woman and man over 50 years of age (1.0-1.2 g/ kg/day with at least $20-25 \mathrm{~g}$ of proteins per meal).

Smoking and alcohol and caffeine: It is recommended to smoking cessation and to limit the consumption of alcohol to no more than 2 units per day. Intake of caffeine by limiting the consumption of no more than 4 cups of coffee per day is recommended.

Prevention of falls: A validated multifactorial assessment of fall risk that evaluates the history of falls and risk of fragility fracture is recommended in patients over 75 years of age, to decide on whom interventions are indicated. A national fall prevention program needs to be structured and implemented in order to prevent institutional and at home falls.
Exercises: A structured exercise program tailored according to the needs and abilities of the individual patient that includes walking, weight training, balance exercises, posture, and flexibility should be incorporated into the routines of the elderly. Physical medicine and rehabilitation can reduce disability, improve physical function, and activities of daily living and lower the risk of subsequent falls in patients with osteoporosis. Fracture Liaison Services which is a coordinated care system that ensures individuals with fractures receive appropriate and multidisciplinary care should implemented nation-wide is recommended.

\section{NSS91}

THE ROLE OF EXERCISES: WHAT TYPE OF EXERCISE, WHEN?

J. Meray $^{1}$

${ }^{1}$ Gazi University Medical Faculty, PMR Department, Ankara, Turkey

Bone is a dynamic tissue and exercise is an important physical stimulus for the development and maintanence of optimal bone mineral density (BMD) and bone strength. In order to expect adaptive responses from bone a-) Loading must be dynamic intermittant rather than static loads. b-) High magnitude loads should be applied rapidly. C-) Loads must be applied in unusual and diverse loading directions and patterns. D-) Few repetitions of loading cycles are preferred if an adequate load intensity is performed. It's also recommended that, the general muscle strength improving principles (spesificity, reversibility, diminished returns etc...) should be considered when designing any exercise program to increase muscle and bone health. Optimal type and dose of exercise session; in other words, magnitude, rate, number of repetitions, frequency, sessions or days per week must be arranged according to patient's general health status and comorbidities. Walking and other forms of aerobic exercise, progressive resistance training. (PRT), high velocity power training, weight-bearing impact exercise. (WBI) and balance mobility training activities are the alternatives of training methods. WBI- exercise applied as 3-5 sets of 10-20 jumps, 4-7 days / week with multidirectional movement patterns are recommended to prevent bone loss in older adults. PRT is also effective to maintain BMD in postmenopausal women (2-3 sets of $8-12$ repetitions at $70-85 \%$ maximal muscle strength) if prescribed at least 2-3 times per week. According to some reports, regular brisk walking has no effect on preventing age-related bone loss although some authors recommend brisk walks at intensities around $75 \%$ or greater of maximum oxygen update with combination of other forms of exercise. Because of some confusing results, our purpose is to evaluate 
appropriate exercise modalities, together with the colleagues in this session.

References (Limited Examples)

1-) Daly RM.et al. Exercise for the prevention of osteoporosis in postmenopausal women; an evidence-based guide to the optimal prescription. Braziion Journal of Physical Therapy 2019; 23(2): 170-180.

2-) Min SK et al. Position Statement: Exercise Guidelines to increase Peak Bone Mass in Adolescents. J Bone Metab 2019;26(4): 225-239.

\section{NSS92}

\section{ARE ORTHOSES AND PHYSICAL MODALITIES EFFECTIVE FOR OSTEOPOROSIS AND/OR OSTEOPOROTIC FRACTURES?}

A. A. Kucukdeveci ${ }^{1}$

${ }^{1}$ Ankara University, Faculty of Medicine, Department of Physical Medicine and Rehabilitation, Ankara, Turkey

Orthoses and physical modalities are administered among the non-pharmacological treatment approaches for osteoporosis. The aim of this presentation is to make an overview of the use and effectiveness of orthoses and physical modalities for people having osteoporosis and/or fractures.

Spinal orthoses are commonly used for the conservative management of osteoporotic vertebral fractures. The goals of orthotic management may be any combination of limiting motion, improving posture, reducing pain and fatigue, and promoting activities and participation. Various types of orthoses, e.g. thoraco-lumbo-sacral, thoraco-lumbal or lumbosacral according to the level of fracture can be prescribed. They can be either rigid, semirigid or flexible. Various systematic reviews evaluated the effectiveness of spinal orthoses. Current evidence is mixed and inconclusive due to the insufficiency of the size and methodological quality of the studies. Weak to moderate evidence suggests the use of semirigid backpack thoracolumbal orhosis for women with vertebral osteoporosis and/or subacute vertebral fractures for improving strength, pain, posture, and quality of life. Weighted kypho-orthosis is also promising as part of the exercise program and postural reeducation.

Physical modalites such as electrical stimulation, pulsedelectromagnetic field therapy and therapeutic ultrasound can also be applied after osteoporotic fractures due to possible positive effects on pain relief, bone metabolism and fracture healing.

Beneficial effects of whole-body vibration on bone mass and musculoskeletal pain have been documented. It has been reported to provide improvement in reducing bone loss in the lumbal spine of postmenopausal women. It may also be used as an intervention for fall prevention.

\section{References:}

1. Parreira PCS, et al. An overview of clinical guidelines for the management of vertebral compression fracture: a systematic review. Spine J. 2017;17:1932-1938.

2. Newman M, et al. Spinal Orthoses for Vertebral Osteoporosis and Osteoporotic Vertebral Fracture: A Systematic Review. Arch Phys Med Rehabil. 2016;97:1013-25.

3. Wang T, et al. Pulsed electromagnetic fields: promising treatment for osteoporosis. Osteoporos Int. 2019;30:267-276. 4. $\mathrm{Ma} \mathrm{C}$, et al. Effect of whole-body vibration on reduction of bone loss and fall prevention in postmenopausal women: a meta-analysis and systematic review. J Orthop Surg Res. 2016;11:24.

\section{NSS93}

\section{NON-PHARMACOLOGIC TREATMENT OF OSTEOPOROSIS IN THE LIGHT OF TWO CASES}

\section{Y. Kirazli ${ }^{1}$}

${ }^{1}$ Ege University School of Medicine, Dept. Physical Medicine and Rehabilitation, Izmir, Turkey

Two cases with osteoporosis, one without any fracture and the other with one vertebral fracture is discussed within this context.

The first case is a 59 year -old woman who was diagnosed as osteoporosis according to the bone densitometry. She doesn't have any clinical or morphometric osteoporotic fracture. Other than anti-hypertensive treatment, she is not on any other medical agent. Her mother has experienced a hip fracture at the age of 78. She smokes 5 cigarettes / day which she was told to quit. She is a social drinker. She is determined not to follow a diet rich in calcium although the dietary intake is below $700 \mathrm{mg} /$ day. Therefore, Ca supplementation was given in addition to the bone protective therapy she has been receiving. She was recommended not to exceed 500-600mg per dose. She was told to have 800 IU of vitamin D3 on a daily basis as the circulating concentration of $25(\mathrm{OH}) \mathrm{D}$ was $35 \mathrm{ng} /$ $\mathrm{mL}$. Exercise with weight, balance and resistance load was recommended, to improve mobility, strength and physical performance.

The other patient is a 75 year-old lady who has fractured T12 vertebra when she bent down to take a key which was on the floor. After a short period of immobilisation, the patient was carefully remobilised with an orthesis and the pain gradually subsided with analgesic treatment. As the circulating concentration of $25(\mathrm{OH})$ D3 was $9 \mathrm{ng} / \mathrm{mL}, 50$ 000 IU was given orally weekly for 8 weeks as a loading dose and followed by a maintenance dose orally. Calcium intake of 1000 to $1200 \mathrm{mg}$ daily, preferably through nutritional intake was suggested. She is also a heavy coffee consumer. She is on a diet with low quantities of protein. Maintenance of an adequate protein intake is important for the preservation of musculoskeletal function. 
A validated multifactorial assessment of fall risk that evaluates the history of falls and risk of fragility fracture was applied. Then, hip pads were also prescribed as she had a high risk of falls. Specific exercises for this patient profile is also discussed.

The recommendations given to these two cases address integrating interventions to modify their lifestyle, mainly calcium and vitamin $\mathrm{D}$ intake, and exercise. Other recommendations include maintaining adequate protein intake, identification and treatment of risk factors for falls, and limiting the consumption of coffee, alcohol and tobacco.

\section{NSS94}

ASSOCIATION OF BODY COMPOSITION WITH DISEASE ACTIVITY IN RHEUMATOID ARTHRITIS M. Radic ${ }^{1}$

${ }^{1}$ Division of Rheumatology and Clinical Immulogy, Center of excellence for Systemic Sclerosis Ministry of Health Republic of Croatia, School of Medicine in Split, University Hospital Centre Split, Split, Croatia

Rheumatoid arthritis (RA) is a chronic form of inflammatory arthritis characterized by multiple joint involvement and significant disability. The term "body composition" refers to the quantification of the different structural components of the human body, which can be estimated at the atomic, molecular, cellular, organ-tissue and whole body levels. Several studies showed that RA is associated with considerable changes in body composition, lipid profile, adipokines and insulin sensitivity. Overweight and obesity are increasing the risk of metabolic, neoplastic and cardiovascular diseases, but can be considered states of inflammatory activation, since the hypertrophic adipocytes of individuals with body compositions of these types are activated and release a number of soluble mediators known as adipocytokines or adipokines. The most important adipokines in terms of their proinflammatory activity are leptin, visfatin, interleukin 1 and tumor necrosis factor $\alpha$. The latter 2 cytokines are relevant in the pathogenesis RA, as they are crucial for the onset and persistence of the destructive synovitis that characterize this disease. Several studies have evaluated the association between changes in body composition in terms of measurements of overweight or obesity and the level of disease activity in RA patients are contradictory. The reasons for this controversy very probably lie in the heterogeneity or weakness of the evaluation tools used both for body composition and the level of disease activity associated with RA, as well as weaknesses in the design and quality of the sample selection. The modulation of RA clinical status by body fat mass is suggested because a significant association was found between body mass index (BMI) and inflammatory activity in those reports with a trend toward higher statistical power. The body composition is already altered in patients with early RA with more fat and less lean mass, with or without an increase in the BMI. Various studies describe the association between high BMI categories and poorer clinical outcomes, a lower chance for remission, and a higher probability of comorbidity but less radiographic joint damage. Body compositions such as body fat mass and skeletal muscle mass are significantly associated with pain and disability in RA patients. The relationship between body composition and clinical activity in RA requires be approached with further studies with higher methodological quality.

\section{NSS95 \\ RELATIONSHIP OF BODY COMPOSITION WITH OSTEOPOROSIS}

T. Vlak ${ }^{1}$

${ }^{1}$ Department of Rehabilitation Medicine and Rheumatology, University Hospital Center Split, Split, Croatia

Osteoporosis is a skeletal disease characterized by low bone mass and microarchitectural deterioration of bone tissue with a consequent increase in bone fragility and susceptibility to fracture. Osteoporosis was defined by the World Health Organization (WHO) in 1994 as a bone mineral density of less than 2.5 standard deviations below the sex-specific young adult mean and this characterization has been adopted globally. The term sarcopenia was first coined in 1989 by Irwin Rosenberg who used it to define/describe the loss of muscle mass with age and it is associated with increased rates of disability, poor mobility, frailty and hospitalization. Since then it has been known that muscle function, in addition to muscle mass, is necessary to describe sarcopenia. Osteoporosis and sarcopenia are common diseases in older age and are associated with significant morbidity and mortality. They represent two major health problems with an increasing prevalence in the elderly population. The correlation between these diseases has been widely reported, leading to the development of the term "osteosarcopenia" which is used to diagnose those patients suffering from both diseases. A universal definition was established for osteoporosis in 1994. but for sarcopenia there is still ongoing debate regarding how best to define it. Human body composition measurements are objective methods of nutritional assessment and area of interest to health professionals. Despite many clinical trials and epidemiological studies, we still didn't define how changes in human body composition affect clinical and functional status in both patients with osteoporosis and sarcopenia. Reasons for that are often found in poorly available diagnostic methods and low motivation of health professionals in relationship of body composition and osteoporosis. CT and MRI represent the gold standard for evaluating body composition (BC), but are costly and time-consuming. Bioelectrical impedance analysis (BIA) is emerging as a popular alternative to DXA due to its 
easy installation and superior cost-effectiveness. BIA measures tissue impedance by circulating a weak alternating current through the body.

Using BIA, we tried to define the relationship of body composition in patients with osteoporosis during the pilot study in cohort of Croatian patients with osteoporosis.

\section{NSS96 \\ IMPACT OF DISABILITY ON BODY COMPOSITION IN MULTIPLE SCLEROSIS}

O. Polašek ${ }^{1}$, M. Radic ${ }^{2}$

${ }^{1}$ Department of Public Health, University of Split School of Medicine, Split, Croatia, ${ }^{2}$ Division of Rheumatology and Clinical Immulogy, Center of excellence for Systemic Sclerosis Ministry of Health Republic of Croatia, School of Medicine in Split, University Hospital Centre Split, Split, Croatia

Multiple sclerosis (MS) is a chronic disease of the central nervous system, more prevalent in women than in men, leading to impairment of motor function and cognitive dysfunction with deterioration of body composition. Significant differences in body composition between body mass index-matched men with and without MS have been reported, although this was not observed in women. According to recent research cognitive function does not correlate to body composition parameters in MS patients. Disability and inactivity varies widely between four different clinical profiles: clinically isolated syndrome, relapsing-remitting MS (RRMS), primary and secondary progressive MS. Impact on body composition has been mostly researched in RRMS. More disabled MS patients present with higher body fat and lower bone tissue content and density than those with milder disability. Physical inactivity in MS patients is associated with changes in body composition. Nondrug-based symptomatic treatment approach in MS encompasses physical therapy with positive effects that include symptom reduction and enhancement of mobility, however there have been contrary results on the impact of body composition. Matusik et al. found that physiotherapy had a significant impact on functional mobility and it was related to body composition. ${ }^{1}$ Whereas, Silveria et al. did not find a correlation between symptoms and body composition in MS patients that underwent physical activity intervention. ${ }^{2}$ Furthermore, pilates has not been shown to impact body composition. High intensity exercise therapy in milder patients seems to have an effect on fat mass, fat percentage and fatfree mass over time. Further research is needed to explore differences in body composition between genders and MS clinical profiles, and develop potential strategies to address changes in body composition in MS.

\section{References}

1. Matusik E, et al. Medicina (Kaunas). 2019;55(12):773.

2. Silveira SL, et al. Rehabil Psychol. 2019;10.1037/ rep0000292.

\section{NSS97}

\section{OSTEOSARCOPENIA}

I. Kostoglou-Athanassiou $^{1}$

${ }^{1}$ Department of Endocrinology, Asclepeion Hospital, Voula, Athens, Greece

In older persons, the combination of osteopenia/osteoporosis and sarcopenia - known as osteosarcopenia - has been proposed as a subset of frailer individuals at higher risk of institutionalization, falls, and fractures. Osteosarcopenic patients have very particular clinical, biochemical, diagnostic, and functional characteristics that could be identified in clinical practice.

In this presentation, a clinical definition of osteosarcopenia aiming to describe the clinical, functional, and biochemical features that are unique to these patients will be presented. The use of imaging combined with functional assessments for the diagnosis of osteosarcopenia will be discussed. We will go over the translational aspects of sarcopenia and osteoporosis research and highlight expected outcomes from different interventions for both conditions. In addition, new therapies targeting both muscle and bone are being developed.

\section{NSS98}

NEUROGENIC OSTEOPOROSIS DUE TO SARCOPENIA IN POST POLIOMYELITIS PATIENTS M. Lissens ${ }^{1}$

${ }^{1}$ Orthopedics, Physical and Rehabilitation Medicine,Thomas More University College and KU Leuven, Leuven, Belgium

Muscle mass, -strength and -density, sarcopenia, exercise and physical activity are correlated with bone mass, bone density and osteoporotic fractures. Bone and muscle decay and dysfunction are seen in ageing, but in neurological disorders with muscular atrophy, bone loss can be seen also at younger ages. The pathophysiology of osteosarcopenia in neurological diseases is complicated. One factor is unloading of affected skeletal muscle, and consequently deconditioning which occurs in any muscle that is not active.

In 30 subjects with post poliomyelitis syndrome (mean age of 56,3 years) femoral neck and lumbar spine bone mineral density (BMD) were measured using dual X-ray absorptiometry Low femoral and lumbar bone mass was found in $80 \%$ and $63 \%$, respectively. Six patients had osteoporotic femoral BMD-scores and 3 ( 1 man and 2 women) had osteoporotic lumbar BMD. Femoral and lumbar osteopenia had 18 and 16, respectively. The lowest BMD scores were present in patients 
with visible and clear lower limb muscle atrophy. The correlation found between low muscle mass and BMD, can be explained by neurogenic bone loss, both due to muscle atrophy and disturbed regulation of bone by the central nervous system.

\section{NSS99}

\section{OSTEOSARCOPENIA SCHOOL}

\section{Y. Dionyssiotis $^{1}$}

${ }^{1} 1$ st Physical Medicine \& Rehabilitation Department, National Rehabilitation Center EKA-KAT, Athens, Greece

Finally, the new holistic concept called Osteosarcopenia school will be presented. Preventive measures and therapeutic interventions that can benefit both muscle and bone simultaneously will be analyzed. This new concept is based on counselling and education of patients as part of a rehabilitation program. Includes a specific pathway to stop the vicious cycle, not only of social isolation, but also of falls and fractures in this population, which are all leading to disability. Rehabilitation uses interventions such as counselling and re-education on how to prevent falls. According to the concept, the focus is mainly shifted on muscle strengthening, balance improvement and reducing all causes of instability (i.e. neurogenic, iatrogenic etc.). To this end, specific exercise programmes combined with a review of drugs, is essential in these patients. Re - education includes interventions targeting to increase functionality of the subjects through social reintegration and improvement in activities of daily living.

\section{NSS100}

\section{GENERAL PROFILE OF THE BUTTERFLY PARTICIPANTS}

$\underline{\text { A. Debain }}^{1}$, V. Knoop ${ }^{1}$, A. Costenoble ${ }^{1}$, S. Vermeiren ${ }^{1}$, R. Vella Azzopardi ${ }^{1}$, I. Bautmans ${ }^{1}$. On behalf of the Gerontopole Brussels Study group. https://fria.research.vub.be/en/ butterfly-study

${ }^{1}$ Frailty in Ageing Research department (FRIA), Vrije Universiteit Brussel (VUB), Brussels, Belgium

Objectives: Longitudinal studies have shown that with aging most individuals tend to develop a chronic low-grade inflammatory profile (CLIP) in which chronic diseases may play an important modifying role, and that such a state is a strong risk factor for frailty and death. (1) Our aim is to identify which amount of health burden is predictive to frailty status as those elderly might profit from early interventions.

Materials and methods: 494 participants of the "BrUssels sTudy on The Early pRedictors of FraiLtY" (BUTTERFLY) were categorized according to health status: A1(completely healthy), A2 (Healthy but lifestyle-related risk), B1 (Only
Musculoskeletal comorbidity), B2 (Non-cardiovascular comorbidity) $\mathrm{C} 1$ (arterial hypertension) $\mathrm{C} 2$ (Cardio-vascular comorbidity) D (Inflammatory pathology or antiinflammatory drugs). Frailty status was assessed by the Groningen Frailty Indicator, the Rockwood Frailty Index and the adapted version of the Fried Frailty Index. We distinguished 3 groups: robust $(\mathrm{R}, \mathrm{n}=218)$, physically prefrail $(\mathrm{PF}, \mathrm{n}=193)$ and frail $(\mathrm{F}, \mathrm{n}=77)$.

Results: After dividing for sex: Age, CRP-level, smoking history, medication/alcohol -intake, MMSE and Rockwood Score were evaluated. $\mathrm{F}$ men had a higher $(\mathrm{p}=0,01)$ medication intake $(5 \pm 3)$ than $\mathrm{PF}(4 \pm 3)$ and $\mathrm{R}$ $(3 \pm 2)$. The same results for medication intake $(\mathrm{p}<0,001)$ between $\mathrm{F}(5 \pm 3), \mathrm{PF}(2 \pm 5)$ and $\mathrm{R}$ women $(3 \pm 2)$ was detected. When divided in to health categories we observed a higher number of $\mathrm{C} 2$ and $\mathrm{D}$ in $\mathrm{F}$ men $(50 \%$ \& $12 \%)$ when compared to $\mathrm{R}(40 \%$ \& $3 \%)$ and $\mathrm{PF}(30 \%$ \& $11 \%)$ peers. In F elderly woman C1 (47\%) was more prevalent than in PF (29\%) and R (34\%).

Conclusion(s): Our cross-sectional data suggests a higher prevalence of arterial hypertension, cardiovascular events and chronic inflammatory conditions in frail elderly compared to robust and prefrail peers.

\section{NSS101}

\section{A NEW BIA EQUATION FOR DETERMINING APPENDICULAR LEAN MASS IN COMMUNITY- DWELLING PERSONS AGED 80+}

J. Van den Broeck ${ }^{1}$, S. Vermeiren ${ }^{2}$, D. Beckwee ${ }^{2}$, R. Vella Azzopardi $^{2}$, I. Beyer ${ }^{2}$, V. Knoop ${ }^{2}$, B. Jansen ${ }^{3}$, S. Delaere ${ }^{2}$, A. Antoine $^{2}$, I. Bautmans ${ }^{2}$, A. Scafoglieri ${ }^{1}$. On behalf of the Gerontopole Brussels Study group. https://fria.research.vub. be/en/butterfly-study

${ }^{1}$ Experimental Anatomy, Vrije Universiteit Brussel (VUB), Brussels, Belgium, ${ }^{2}$ Frailty in Ageing Research department (FRIA), Vrije Universiteit Brussel (VUB), Brussels, Belgium, ${ }^{3}$ Department of Electronics and Informatics (ETRO), Vrije Universiteit Brussel (VUB), Brussels, Belgium

Objective: We aimed to evaluate the agreement between dual energy X-ray absorptiometry (DXA) and BIA equations to determine lean mass in the oldest old (80+), as well as their suitability to identify sarcopenia.

Materials and methods: 174 well-functioning persons (mean age: $83,3 \pm 3,0$; mean BMI: $26,8 \pm 3,5$ ) were included. 1) Appendicular lean mass (ALM) was predicted using BIAbased equations available in literature (Kyle et al. 2003, Sergi et al. 2015, Scafoglieri et al. 2017), and compared to DXA outcomes 2) A new ALM-formula was generated suitable for this population 3) Prevalence and agreement for sarcopenia based on BIA-equations was calculated (compared to the standard reference cut-offs for proposed by EWGSOP) 
Results: 1) Literature-based BIA equations systematically overestimate ALM 2) The new prediction formula that we propose for the $80+$ is: $A L M=0,827+(0,19 *$ Impedance Index $)+(2,101 *$ Sex $)+(0,079 *$ Weight $) ; \mathrm{R}^{2}=0,888 ; \mathrm{SEE}=$ $1,450 \mathrm{~kg} .3$ ) BIA equations underestimate sarcopenia regardless the EWGSOP cut-off used. Sarcopenia classification based on our new BIA equation for ALM shows better agreement with DXA $(k \geq 0,454)$ compared to literature-based BIA equations $(\mathrm{k}<0,368)$.

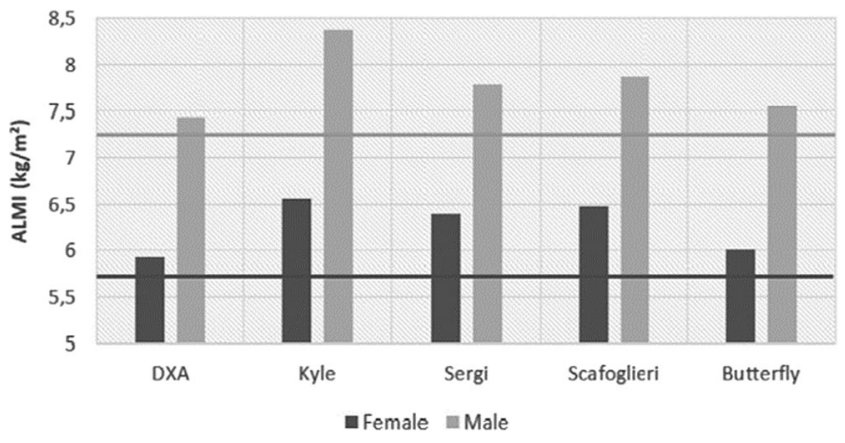

Fig. 1 ALM index according to DXA and BIA-prediction formulas. The horizontal lines represent EWGSOP cut-offs for sarcopenia proposed by Delmonico et al. 2007 (Men < $7.25 \mathrm{~kg} / \mathrm{m}^{2}$, Women $<5.67 \mathrm{~kg} / \mathrm{m}^{2}$ )

Conclusions: Despite the high correlation between both methods, literature-based BIA equations consistently overestimate ALM compared to DXA in persons aged 80 and over. We propose a new equation for ALM, reaching higher agreement with DXA and thus improving the accuracy of BIA for this specific age group.

\section{NSS102}

IMMUNOSENESCENCE SIGNATURES IN PREFRAILTY

R. Njemini ${ }^{1}$, H. Cao Dinh ${ }^{1}$, I. Beyer ${ }^{1}$, T. Mets ${ }^{1}$, O. O. Onyema $^{1}$, L. N. Forti ${ }^{1}$, W. Renmans ${ }^{2}$, S. Vander Meeren ${ }^{2}$, K. Jochmans ${ }^{2}$, S. Vermeiren ${ }^{1}$, R. Vella-Azzopardi ${ }^{1}$, I. Bautmans ${ }^{1}$. On behalf of the Gerontopole Brussels Study group. https://fria.research.vub.be/en/butterfly-study
${ }^{1}$ Frailty in Ageing Research department (FRIA), Vrije Universiteit Brussel (VUB), Brussels, Belgium, ${ }^{2}$ Laboratory of Hematology, Universitair Ziekenhuis, Brussels, Belgium

Objective: In the present study, we sought to investigate the association between markers of cellular immunosenescence and the risk of pre-frailty in community dwelling older persons.

Material and methods: One hundred and seventy-three older persons aged 80 years and over, who presented no active pathology, were enrolled in the study after informed consent. Pre-frailty was defined -using the Fried et al. criteria- as the presence of 1 or 2 out of the following frailty indicators: unintentional weight loss, self-reported exhaustion, weakness, slow walking speed, and low physical activity. The surface markers of senescence were determined using flow cytometry. Cytomegalovirus (CMV) serostatus and serum IL-6 were measured using Architect iSystem and Luminex, respectively. Results: The pro-inflammatory cytokine IL-6 was significantly higher in pre-frail compared to robust individuals $(p<0.001)$. When considering the entire cohort, there were no significant differences in the expression of senescent surface markers, CD4-/CD8+ ratio or CMV-seropositivity between pre-frail and robust individuals. However, in the CMV-seronegative population as well as the CD4-/CD8+ ratio $>4$ group, we found a significantly higher proportion of the highly differentiated memory and senescence-like phenotypes and a lower proportion of the naive subset in pre-frail compared to robust (all $\mathrm{p}<0.05$ ).

Conclusion: These findings indicate that the presence of prefrailty is independent of CMV infection in very old persons. Moreover, higher concentrations of IL-6, age and sex were independently predictive of pre-frailty. Whether IL-6 might facilitate the identification of people at risk of developing pre-frailty deserves further investigation

Publisher's Note Springer Nature remains neutral with regard to jurisdictional claims in published maps and institutional affiliations. 